University of Navarra

\title{
PRICING IN A DUOPOLY WITH A LEAD TIME ADVANTAGE
}

\author{
Víctor Martínez de Albéniz ${ }^{1}$
}

${ }^{1}$ Professor, Operations Management and Technology, IESE

IESE Business School - University of Navarra

Avda. Pearson, 21 - 08034 Barcelona, Spain. Tel.: (+34) 932534200 Fax: (+34) 932534343

Camino del Cerro del Águila, 3 (Ctra. de Castilla, km 5,180) - 28023 Madrid, Spain. Tel.: (+34) 913570809 Fax: (+34) 913572913

Copyright ${ }^{\circledR} 2007$ IESE Business School. 


\title{
Pricing in a Duopoly with a Lead Time Advantage
}

\author{
Victor Martínez-de-Albéniz \\ IESE Business School, University of Navarra, \\ Av. Pearson 21, 08034 Barcelona, Spain \\ valbeniz@iese.edu
}

Submission: July 6, 2006

\begin{abstract}
We analyze the price competition between two suppliers offering two different lead times and two different prices to a buyer. The buyer chooses its inventory replenishment policy in order to minimize its infinitehorizon average cost. In essence, the fast and expensive supplier is used only in emergencies, while the slow and cheap supplier receives the bulk of the orders. Thus, despite a higher price, the fast supplier is able to capture a part of the buyer's orders. We analyze the price competition between the asymmetric suppliers, where the market share of each supplier is derived from the buyer's inventory problem. We find equilibria that differ significantly from the Bertrand price-only competition. In particular, for some cost parameters, the fast supplier is able to charge a premium for faster delivery, and stay in business even with a higher production cost. We obtain in some cases closed-form formulas for the price difference in equilibrium. Hence, our results show that high cost suppliers may not be driven out of business if they can offer fast delivery.
\end{abstract}

\section{Introduction}

Globalization of the economy has had tremendous consequences for many companies around the world. The major effects have been observed in manufacturing, e.g., textiles or electronics, where a large volume of production has moved from high cost countries, such as the United States, Germany or France, to low cost regions, such as China, Vietnam, or Eastern European countries.

Of course, for companies based in higher cost areas such as Western Europe or the United States, facing new competition from low cost countries has been a challenge difficult to overcome. As a result, many of these firms have moved their own facilities to low cost countries as it seemed the only way of remaining competitive.

However, by moving to low cost countries, these companies have also given up an important competitive attribute: typically, they have moved from locations close to the final market, to far-away countries, which are weeks away from the customers. Implicitly, these companies have assumed that the lead time advantage was not as valuable as the cost advantage. 
These actions seem to imply that in the new competitive landscape, cost is the only important attribute to consider. The economics literature has developed some game-theoretic price competition models that support this view of the world. Most relevant is the Bertrand price competition framework, see Tirole [17] or Vives [19] for references. In this setting, two firms compete in price for a market demand. When the two firms have identical costs, the only equilibrium is that both firms price at marginal cost, share the total demand, and thus make zero profits. This is the so-called Bertrand paradox. A more realistic use of this model is to examine the asymmetric case, when one firm has lower costs than the other. Here, the most competitive supplier captures the market, at an equilibrium price equal to the cost of the less competitive supplier. This is what seems to be observed in the offshoring trend. These models can be extended to take lead time into account, by adding a cost penalty to the slow supplier. However, by doing this, the equilibrium result is similar to the Bertrand outcome: one supplier captures all the market and drives the least competitive suppliers out of business.

In some industries, however, we can observe suppliers located in high cost countries coexisting with low-cost competitors. In these situations, the lead time advantage must certainly be considered, as it allows companies to serve the market better. For instance, fast-retailing companies such as Zara have demonstrated that sourcing close to the market can provide a crucial competitive advantage. Zara can achieve very fast response to market trends by using suppliers in Spain, Portugal and Northern Africa. Zara's suppliers are probably more expensive than competitors located in Asia, but their short lead time makes them Zara's first choice.

Other companies, such as the German retailer Adidas, also take advantage of lead time in their supply. Adidas may work with two suppliers: one in China and another one in Germany. Having two suppliers helps Adidas react to demand variability better (with the reactivity of the German supplier) and obtain good prices (by giving volume to the Chinese supplier). This example shows that the German supplier, Trigema [9], can be competitive offering fast service and high price.

Obviously, such a situation cannot be explained with a Bertrand-like competition model, where the winner takes all. Indeed, Adidas' case shows that it is possible to obtain the benefits of both low cost and high flexibility by using multiple sourcing. Concentrating supply in a single source limits the production flexibility that the buyer can obtain: by using a unique supplier, the buyer commits to a single production technology, which is typically cheap but rigid, or expensive and flexible. To alleviate this effect, many companies resort to dual sourcing. Like Adidas, the buyer can then use the fast and expensive supplier in emergencies, while the slow and cheap supplier receives the bulk of the orders. Under this buyer's 
behavior, it is clear that, despite a higher price, the fast supplier is able to capture a part of the buyer's orders. Faster lead time hence provides an opportunity for high cost suppliers to be competitive and stay in business.

The purpose of this paper is thus to provide a model of supplier competition where this lead time advantage is properly taken into account. This requires using a dual sourcing inventory management model. We analyze explicitly the buyer's inventory management policy to characterize the market share that the fast supplier can capture, despite a higher price. We then turn to analyzing the duopoly pricing game between fast and slow suppliers. We ask the following questions. First, can the fast supplier achieve a positive share of the market even though it has higher cost? Second, in equilibrium, what is the price premium, if any, that it can charge, compared to the slow supplier?

Our model consists of an infinite-horizon periodic-review inventory replenishment problem. The buyer faces a stochastic i.i.d. demand at each period, and can replenish its inventory by placing orders with two suppliers, each offering a different lead time and a different price to the buyer. We assume that there is no fixed cost associated with ordering from any of the two suppliers. The buyer minimizes its long-run average cost by placing appropriate orders with each supplier. This minimization results in a splitting of the market between the suppliers.

Knowing this splitting, each supplier chooses a static price for the component that maximizes its longterm average profit, given its cost and its lead time quotation. We analyze the pure-strategy equilibrium pricing strategies of the suppliers.

Of course, we could give the suppliers the freedom to adjust prices as a function of the buyer's current stocking position. In a situation of potential shortage, the fast supplier, being the only supplier that can save the buyer from a stock-out, would raise the price of the component and use its temporarily increased negotiation power. Studying this behavior would require a complex model that monitors the quality of buyer-supplier relationships. Instead, we choose to study a static price game, where it is assumed that suppliers do not take advantage of the buyer's situation. In practice, this may correspond to long-term price agreements between suppliers and buyer. Within this framework, our model is able to capture the intrinsic advantages of a fast-response supplier.

We find that for some cost parameters, the asymmetric Bertrand equilibrium remains the only equilibrium of our game. However, we also characterize situations where the fast supplier captures a positive share of the market. Our model thus identifies competitive equilibria that are very different in nature to the ones identified by traditional price-only competition. Finally, we find that in some cases, no equilibrium exists. 
In any case, we observe that the demand distribution has a direct impact in the nature of competition.

The paper is organized as follows. In Section 2, we review the existing literature. We present the model in Section 3. We first analyze the buyer's inventory problem in Section 4. Section 5 presents general equilibrium results for general market splitting functions. These are applied to two cases. First, in Section 6, we analyze the duopoly competition when the lead time difference is equal to the review period, and obtain closed form results when the lead times are 0 and 1. Second, in Section 7, we develop an approximated market splitting function and characterize the different equilibrium cases. Finally, in Section 8, we summarize our results and discuss possible extensions of the model.

\section{Literature Review}

Our model considers competition between suppliers in a context of inventory management, where price together with lead time is taken into account.

A first stream of literature related to this work considers sourcing with multiple suppliers taking into account price and another attribute, such as quality, yields or credit risk. Minner [12] reviews several motivations for supplier diversification. The objective is to understand how this additional attribute affects the buyer's profit or utility function. These models typically derive appropriate operating policies, by identifying the ordering policies that the buyer should use with each supplier. Specifically, when this is applied to the case of dual sourcing, the key question for the buyer is how to split orders between the suppliers.

One of the main attributes that has been considered in the literature is supplier yield. That is, when suppliers are not fully reliable and deliver only a fraction of their orders, then supplier diversification can be beneficial in order to reduce yield uncertainty. This approach is motivated by quality problems or response-time uncertainties. Gerchak and Parlar [8], Anupindi and Akella [2], and Parlar and Wang [15] discuss the optimal diversification policy for the buyer, i.e., its optimal replenishment strategy. In a similar line, when there is a chance that a supplier goes out of business, using dual sourcing reduces the risk of a supply disruption, see Tomlin [18], Federgruen and Yang [5] and Babich et al. [3].

Closest to this paper is a set of papers that consider lead time and price attributes, see Allon and Federgruen [1] and references therein. These papers use queuing theory to model lead time. Our paper follows an approach similar to the papers presented above, with the difference that we specify lead time explicitly (and not as the outcome of server congestion). This is more realistic for situations where lead time is related to transportation. There, capacity is seldom restrictive and uncertainty in transportation 
time is minimal. The focus is also different. We start by determining the buyer's behavior when facing two competing suppliers with different price and lead time combinations, and use it to analyze the competitive interaction between the suppliers through price.

In addition to the papers cited above, we make use of the literature of inventory management with multiple procurement modes. It is well-known, from Fukuda [7], that when the lead time difference between the suppliers is equal to 1 , then a double base-stock policy is optimal for the buyer. However, when the lead time difference is greater than 1 , the optimal replenishment policies do not exhibit the base-stock property anymore, see Feng et al. [6] and Whittemore and Saunders [20]. In this paper, we assume that the buyer uses a double base-stock policy, that, despite being sub-optimal, is commonly used in double sourcing practices. Intuitively, by using a static base-stock policy, the bulk of the procurement comes from the distant and cheap supplier, and only when the inventory level is dangerously low (i.e., below the corresponding base-stock target), the fast and expensive supplier is called in. A similar heuristic approach has been extensively used in the literature: impose a given simple policy, and find the corresponding optimal or near-optimal parameters, see for instance Moinzadeh and Nahmias [13], Moinzadeh and Schmidt [14] or Tagaras and Vlachos [16].

Finally, our work is related to pricing too. In a single period monopoly setting, the optimal pricing policy has been studied in Lariviere and Porteus [10], where a supplier is selling to a newsvendor. Introducing competition complicates the nature of the analysis, and uses more intensively game theory. Our work is thus related to the huge body of research in economics concerned with oligopoly pricing, and in particular duopoly pricing. The book by Vives [19] reviews most models in that area, from Bertrand to Cournot competition. This paper falls within the category of price-competition models with asymmetric firms. In the economics literature, the Bertrand model with asymmetric costs is the closest work to our model. The main difference is that in our case, the demand is split between the two suppliers that charge different prices. In contrast, in the Bertrand model, when a firm charges a higher price than its competitor, then its market share is zero.

\section{The General Model}

Consider a buyer that needs to purchase a component for the final product, in a infinite-horizon periodicreview environment. Demand for the component is stochastic. Customers arriving at time $t=1, \ldots, \infty$ are served from stock, or, if on-hand inventory is not sufficient, unserved items are back-ordered. We denote by $D_{t}$ the demand from period $t$, and we assume that demands are independent identically distributed and 
within the support $\left[D_{\min }, D_{\max }\right] \subset[0, \infty)$. Let $\bar{F}(q)=\mathbb{P}\left(D_{t} \geq q\right)$ and $\bar{D}$ the average demand. We assume that $\bar{F}$ is twice differentiable, and $\bar{F}^{\prime}=-f$. We denote, for $k \geq 1, \bar{F}_{k}(q)=\mathbb{P}\left(D_{t}+\ldots+D_{t+k-1} \geq q\right)$ and $\bar{F}_{k}^{\prime}=-f_{k}$.

The component may be obtained from two different suppliers, a local supplier, 1, and an offshore supplier, 2. Of course, these two suppliers differ not only by the prices they charge, but also by their lead times. We assume, without loss of generality, that the local supplier has a lead time $L_{1} \geq 0$ while the offshore supplier needs a time $L_{2}>L_{1}$ to serve the buyer. That is, when an order is placed at supplier $i$ at the end of period $t$ (after demand at $t$ is realized), it is received at the beginning of period $t+L_{i}+1$ (before the demand at $t+L_{i}+1$ is realized).

We denote by $p_{1}, p_{2}$ the price that these two suppliers charge for one unit of component. Of course, if $p_{1} \leq p_{2}$, it is clear that the buyer should always source from the cheaper and faster supplier. However, when $p_{1}>p_{2}$, the optimal policy can be to source from both suppliers.

The sequence of events is the following. First, the suppliers set long-run static prices $p_{1}$ and $p_{2}$. Then, the buyer determines its optimal long-run replenishment policy. We are ultimately interested in the price competition between suppliers, and for this purpose, we analyze the buyer's behavior first.

\section{The Buyer's Inventory Problem}

The buyer's objective is to serve demand at minimum long-run average cost. Three factors contribute to cost:

- the cost of items purchased at supplier 1 ;

- the cost of items purchased at supplier 2; and

- the inventory holding and back-ordering cost.

We model the holding and back-ordering cost as a piecewise linear function $h(\cdot)$. Specifically, $h(q)=$ $h_{i n v} \cdot q$, when $q \geq 0$, where $h_{i n v} \geq 0$ is the per-unit holding cost; and $h(q)=-h_{b o} \cdot q$, when $q \leq 0$, where $h_{b o} \geq 0$ is the per-unit back-ordering cost. This choice of cost function is standard in inventory models. As a matter of fact, the analysis could be performed for a more general function $h(\cdot)$, that should be convex.

The supply flow in the chain is modeled as follows. For each time period $t$, we define the inventory position $j, Y_{t}^{j}$, as the total of the inventory on-hand plus all the standing orders due before $t+j$. These 
include all the orders placed before $t$, but not yet received. Thus, $Y_{t}^{0}$ represents the inventory on-hand (negative is there is backlogged demand), $Y_{t}^{1}$ the sum of $Y_{t}^{0}$ plus the arrivals of the next day, and so on.

Hence, as shown in Figure 1, the inventory state at the beginning of time $t$ is

$$
\left(Y_{t}^{0}, \ldots, Y_{t}^{L_{1}-1}, Y_{t}^{L_{1}}, \ldots, Y_{t}^{L_{2}-1}, Y_{t}^{L_{2}}\right)
$$

at this point, demand $D_{t}$ arrives into the system, and the state moves into

$$
\left(Y_{t}^{0}-D_{t}, \ldots, Y_{t}^{L_{1}-1}-D_{t}, Y_{t}^{L_{1}}-D_{t}, \ldots, Y_{t}^{L_{2}-1}-D_{t}, Y_{t}^{L_{2}}-D_{t}\right)
$$

finally, replenishment orders are sent and time advances from $t$ to $t+1$, with state

$$
\begin{aligned}
& \left(Y_{t+1}^{0}, \ldots, Y_{t+1}^{L_{1}-1}, Y_{t+1}^{L_{1}}, \ldots, Y_{t+1}^{L_{2}-1}, Y_{t+1}^{L_{2}}\right) \\
& =\left(\begin{array}{l}
Y_{t}^{1}-D_{t}, \ldots, Y_{t}^{L_{1}}-D_{t}, Y_{t}^{L_{1}+1}+Q_{t}^{1}-D_{t}, \ldots, \\
Y_{t}^{L_{2}}+Q_{t}^{1}-D_{t}, Y_{t}^{L_{2}}+Q_{t}^{1}+Q_{t}^{2}-D_{t}
\end{array}\right),
\end{aligned}
$$

where $Q_{t}^{1}$ and $Q_{t}^{2}$ are the ordering quantities placed in $t$ at suppliers 1 and 2 respectively. We see that when supplier 1 has lead time $L_{1}=0$, this corresponds to replenishment orders that are fulfilled immediately, i.e., before the next demand arrives.

Given the prices $p_{1}$ and $p_{2}$, let $\left(q^{1}\left(Y_{t}\right), q^{2}\left(Y_{t}\right)\right)$ be a Markov replenishment policy, among which we find the optimal one. The average cost of the buyer using that policy can be written as

$$
\begin{aligned}
C\left(q^{1}, q^{2}\right) & =p_{1} \mathbb{E}\left[q^{1}(Y)\right]+p_{2} \mathbb{E}\left[q^{2}(Y)\right]+\mathbb{E} h\left(Y^{0}\right) \\
& =\left(p_{1}-p_{2}\right) \mathbb{E}\left[q^{1}(Y)\right]+p_{2}\left(\mathbb{E}\left[q^{1}(Y)\right]+\mathbb{E}\left[q^{2}(Y)\right]\right)+\mathbb{E}\left[h\left(Y^{0}\right)\right],
\end{aligned}
$$

where $Y$ is the steady-state distribution of $Y_{t}$ using the policy $\left(q^{1}, q^{2}\right)$. Any policy that yields a finite average cost should satisfy $\mathbb{E}\left[q^{1}(Y)\right]+\mathbb{E}\left[q^{2}(Y)\right]=\bar{D}$, for stability. Thus, any candidate for the optimal policy, we must have

$$
C\left(q^{1}, q^{2}\right)=\left(p_{1}-p_{2}\right) \mathbb{E}\left[q^{1}(Y)\right]+p_{2} \bar{D}+\mathbb{E}\left[h\left(Y^{0}\right)\right]
$$

This implies a very important property: the optimal policy will depend only on

$$
\Delta:=p_{1}-p_{2}
$$

the price difference, and not on the prices taken separately.

Let

$$
\alpha_{1}(\Delta)=\frac{\overline{Q^{1}}(\Delta)}{\bar{D}}
$$

the fraction of orders going to supplier 1, i.e., its average market share, under the buyer's optimal replenishment policy. The fraction of orders going to supplier 2 is simply $\alpha_{2}(\Delta)=1-\alpha_{1}(\Delta)$. 


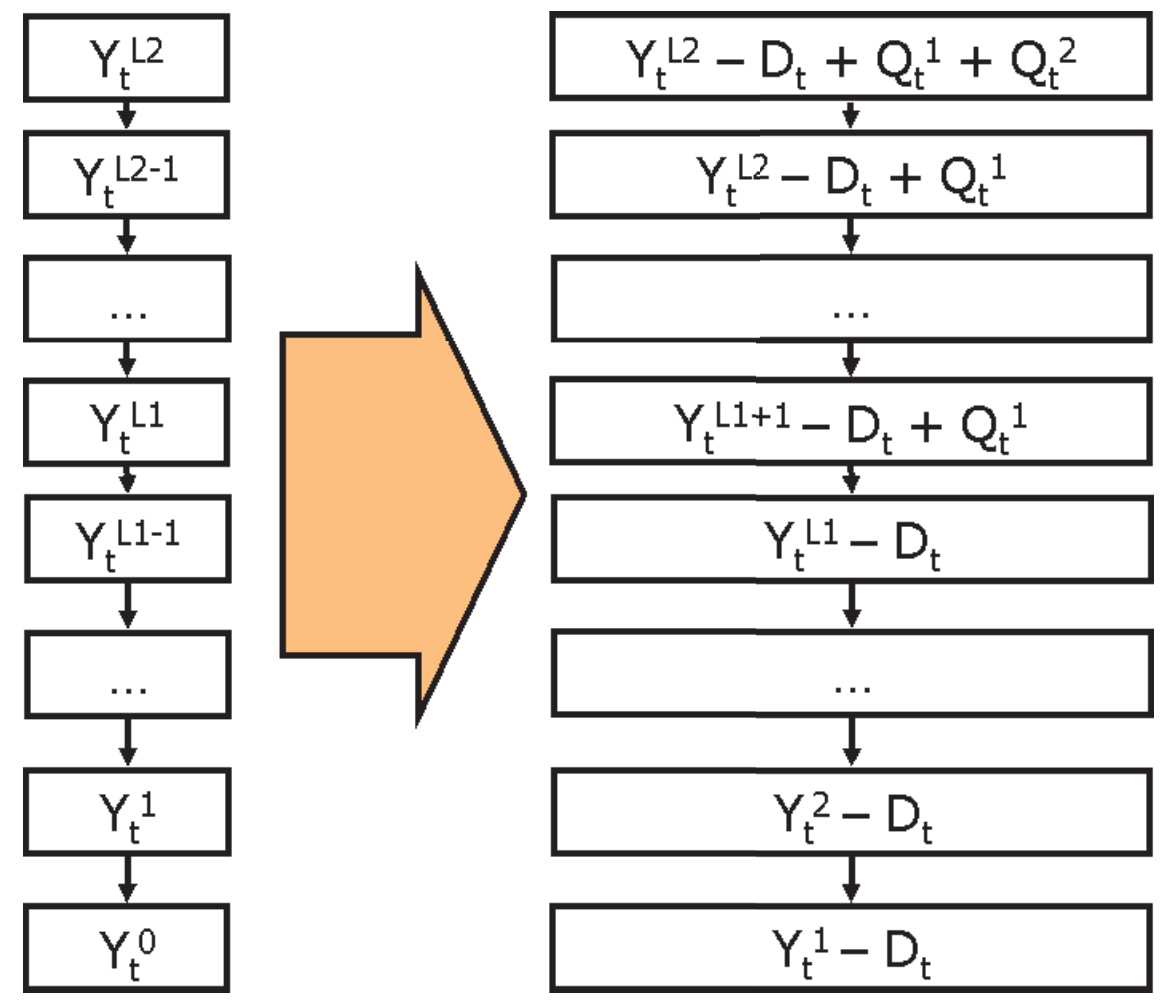

Figure 1: Summary of the inventory position at times $t$ (left) and $t+1$ (right). 
One direct property is that when $\Delta=0$, the buyer should source only from the fast supplier, i.e., $\alpha_{1}(0)=1$. In addition, when $\Delta \geq\left(L_{2}-L_{1}\right) h_{b o}$, the buyer is better off buying from the slow supplier and stocking out, rather than buying from the fast supplier. Thus it should source only from the slow supplier, i.e., $\alpha_{1}\left(\left(L_{2}-L_{1}\right) h_{b o}\right)=0$. In addition, it is clear that $\alpha_{1}$ is non-increasing.

Figure 2 shows an example of the shape of the market share function $\alpha_{1}$, an output of the buyer's inventory problem. We consider a normal demand distribution with mean 1 and standard deviation of 0.3 , inventory and back-ordering cost $h_{i n v}=1$ and $h_{b o}=9$ respectively, and lead times $L_{1}=2$ and $L_{2}=3$. Notice that when $\Delta \rightarrow 0$, the market share of the fast supplier goes to $100 \%$, i.e., $\alpha_{1} \rightarrow 1$.

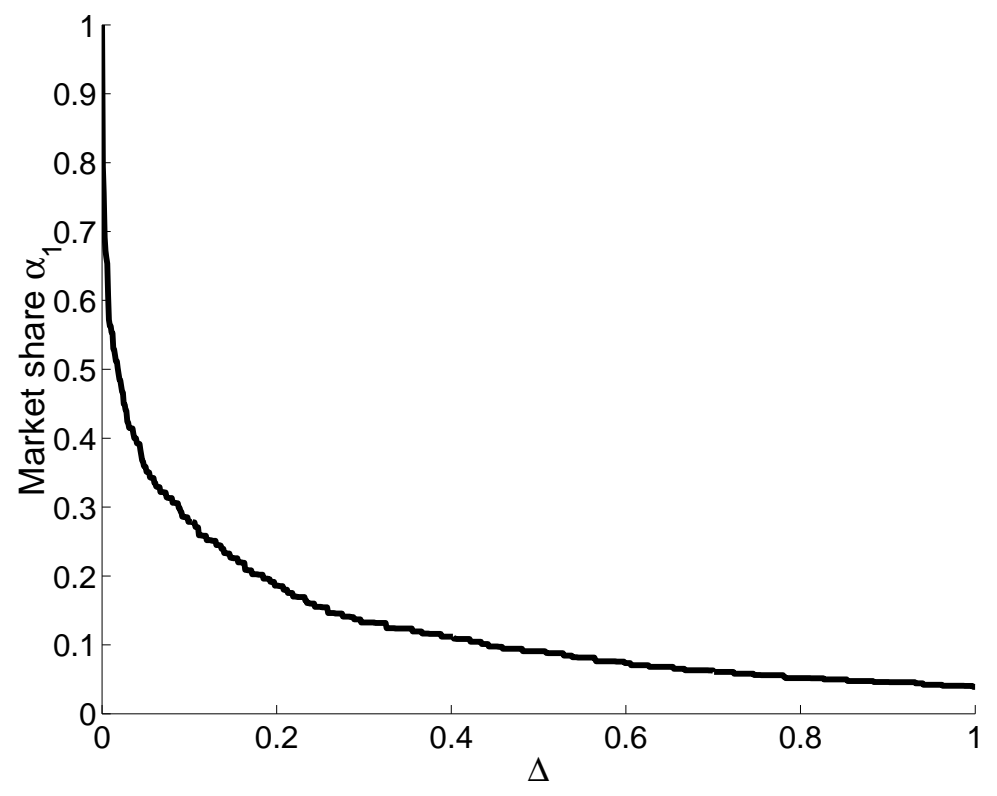

Figure 2: Shape of $\alpha_{1}(\Delta)$ as a function of $\Delta$, for $L_{1}=2$ and $L_{2}=3$.

Notice that for any combination $\left(L_{1}, L_{2}\right), \alpha_{1}(\Delta)$ can only be characterized by solving the buyer's minimization problem. The optimal inventory policy in the general case is known to have a complex structure, see Whittemore and Saunders [20]. However, when $L_{2}=L_{1}+1$, Fukuda [7] first showed that using a double base-stock replenishment policy was optimal. Not only the class of double base-stock policies is optimal when $L_{2}=L_{1}+1$, but also, when $L_{2}>L_{1}+1$, its performance is close to the optimum, as shown in Tagaras and Vlachos [16]. Moreover, its simplicity makes it an attractive candidate for companies to implement. 
The characterization of $\alpha_{1}$ using double base-stock policies is the focus of Sections 6 and 7. However, before analyzing this in detail, we define the duopoly price competition and obtain some results that depend on the structure of $\alpha_{1}$. For this purpose, we focus on the suppliers' strategies.

\section{Duopoly Prices in Equilibrium}

We assume that the suppliers incur a cost $c_{1}, c_{2}$ respectively per unit of component shipped to the buyer. Of course, in order to earn profits, the suppliers declare a price that is higher than their true cost. We compute the profits of each one of the suppliers (we scale it down by the average demand). Supplier 1's profit is hence

$$
\Pi^{1}\left(p_{1}, p_{2}\right)=\left(p_{1}-c_{1}\right) \alpha_{1}
$$

while supplier 2's profit can be expressed as

$$
\Pi^{2}\left(p_{1}, p_{2}\right)=\left(p_{2}-c_{2}\right)\left(1-\alpha_{1}\right)
$$

We study the Nash equilibria of the game in pure strategies. Our model resembles the classical Bertrand price-competition model. In Bertrand's setting, the function $\alpha_{1}$ is such that:

$$
\alpha_{1}(\Delta)= \begin{cases}1 & \text { when } \Delta<0 \\ \frac{1}{2} & \text { when } \Delta=0 \\ 0 & \text { when } \Delta>0\end{cases}
$$

With this demand splitting scheme, equilibrium exists, and is unique such that $p_{1}=c_{1}, p_{2}=c_{1}-\epsilon>c_{2}$ (an $\epsilon$-equilibrium).

In our model, on the other hand, $\alpha_{1}$ is the outcome of the buyer's optimization problem, given the price difference $\Delta=p_{1}-p_{2}$. It can be observed that $\alpha_{1}(0)=1$ and decreases to zero with $\Delta$. The central question here is to determine whether equilibrium exists, and if so, whether it is unique. Of course, the answer to this question depends crucially on the shape of the function $\alpha_{1}$.

Lemma 1 If $\left(p_{1}, p_{2}\right)$ is a Nash equilibrium of the pricing game with $p_{1}>p_{2}$, then it is necessary that

$$
-\frac{d \alpha_{1}}{d \Delta}=\frac{\alpha_{1}}{p_{1}-c_{1}}=\frac{\alpha_{2}}{p_{2}-c_{2}}
$$

together with

$$
2\left(\frac{d \alpha_{1}}{d \Delta}\right)^{2} \geq \alpha_{1} \frac{d^{2} \alpha_{1}}{d \Delta^{2}} \quad \text { and } \quad 2\left(\frac{d \alpha_{1}}{d \Delta}\right)^{2} \geq-\left(1-\alpha_{1}\right) \frac{d^{2} \alpha_{1}}{d \Delta^{2}}
$$


This is simply a reformulation of the first and second-order conditions. Notice, however, that, unless we show concavity of the profit function of each supplier, the second-order conditions do not guarantee that the selected price is a global maximizer of profit. Thus, we need to conduct a more thorough analysis of the profit functions. For this purpose, we identify properties of $\alpha_{1}$ that yield equilibrium results.

Lemma 2 If $\alpha_{1}$ is convex in $\left(0,\left(L_{2}-L_{1}\right) h_{b o}\right)$, then the profit function $\Pi_{2}$ is pseudo-concave in $p_{2} \in$ $\left(p_{1}-\left(L_{2}-L_{1}\right) h_{b o}, p_{1}\right)$.

All the proofs are contained in the appendix.

Lemma 2 shows that the profit of the slow supplier, 2 , is well-behaved when $\alpha_{1}$ is convex. It turns out that this property is satisfied by most usual distributions, see Lemma 7 in the next section. With this property, we can characterize the best-response price $p_{2}$ to a price $p_{1}$.

Lemma 3 When $\alpha_{1}$ is convex in $\left(0,\left(L_{2}-L_{1}\right) h_{b o}\right)$, then the best-response $p_{2}^{*}\left(p_{1}\right)$ has the following characteristics: there are $0 \leq a_{2} \leq e_{2}$ such that

- when $p_{1} \leq c_{2}$, then $p_{2}^{*}\left(p_{1}\right)=c_{2}$;

- when $c_{2}<p_{1} \leq c_{2}+a_{2}, p_{2}^{*}\left(p_{1}\right)=p_{1}-\epsilon$ with $\epsilon$ positive very small;

- when $c_{2}+a_{2}<p_{1} \leq c_{2}+e_{2}, p_{2}^{*}$ is continuous and non-decreasing in $p_{1}$, but with a slope smaller than 1

- when $c_{2}+e_{2}<p_{1}, p_{2}^{*}\left(p_{1}\right)=p_{1}-\left(L_{2}-L_{1}\right) h_{b o}$.

Thus, $p_{2}^{*}$ is non-decreasing, continuous everywhere, with slope between 0 and 1 , except at $p_{1}=c_{2}+e_{2}$, where it jumps up.

We can see that there are four main cases for the slow supplier: either exit the market by pricing higher than the fast supplier; or settle for the deterministic part of demand only, i.e., $\alpha_{2}\left(0^{+}\right)$, which may or may not be zero, by pricing marginally below the fast supplier; or undercut the fast supplier to capture market share; or push the fast supplier out of the market by setting a much smaller price $p_{1}-\left(L_{2}-L_{1}\right) h_{b o}$. The four cases are illustrated in Figure 3 (left).

We can perform a similar analysis for the fast supplier. Here the critical property is having $\frac{1}{\alpha_{1}}$ convex. 
Lemma 4 If $\frac{1}{\alpha_{1}}$ is convex in $\left(0,\left(L_{2}-L_{1}\right) h_{b o}\right)$, then the profit function $\Pi_{1}$ is pseudo-concave in $p_{1} \in$ $\left(p_{2}, p_{2}+\left(L_{2}-L_{1}\right) h_{b o}\right)$.

Lemma 5 When $\frac{1}{\alpha_{1}}$ is convex in $\left(0,\left(L_{2}-L_{1}\right) h_{b o}\right)$, then the best-response $p_{1}^{*}\left(p_{2}\right)$ has the following characteristics: there are $-\left(L_{2}-L_{1}\right) h_{b o} \leq a_{1} \leq e_{1}$ such that

- when $p_{2} \leq c_{1}-\left(L_{2}-L_{1}\right) h_{b o}$, then $p_{1}^{*}\left(p_{2}\right)=c_{1}$;

- when $c_{1}-\left(L_{2}-L_{1}\right) h_{b o}<p_{2} \leq c_{1}+a_{1}, p_{1}^{*}\left(p_{2}\right)=p_{2}+\left(L_{2}-L_{1}\right) h_{b o}-\epsilon$ with $\epsilon$ positive very small;

- when $c_{1}+a_{1}<p_{2} \leq c_{1}+e_{1}, p_{1}^{*}$ is continuous in $p_{2}$, with a slope smaller than 1 (may be decreasing);

- when $c_{1}+e_{1}<p_{2}, p_{1}^{*}\left(p_{2}\right)=p_{2}$.

Thus, $p_{1}^{*}$ is continuous everywhere except at $p_{2}=c_{1}+e_{1}$ possibly, where it jumps down. When $\alpha_{1}$ is continuous at $\Delta=0$, then $p_{1}^{*}$ is continuous everywhere.

Similarly as with Lemma 3, we obtain four cases. These are illustrated in Figure 3(right).
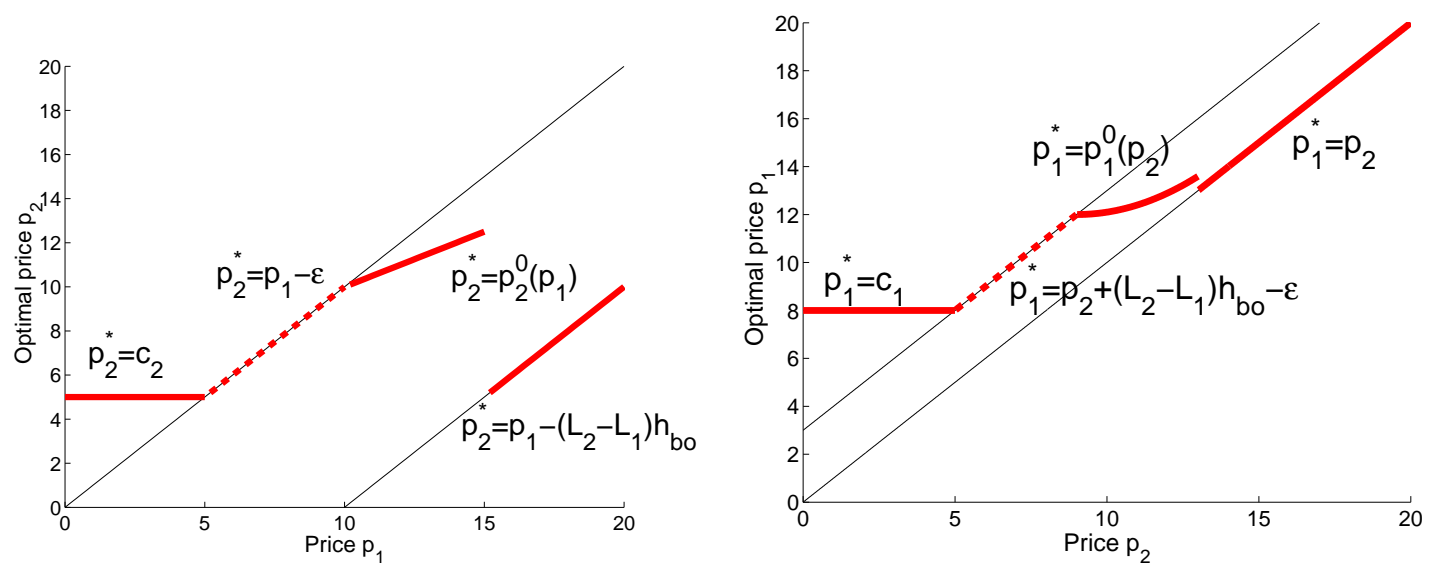

Figure 3: Shape of the best-response functions $p_{2}^{*}\left(p_{1}\right)$ (left) and $p_{1}^{*}\left(p_{2}\right)$ (right). Notice that the intermediate part, where $p_{i}^{*}=p_{i}^{0}$ continuous, is here increasing, but it is not necessarily so for $p_{1}^{*}$ as we see in Section 6 .

However, it turns out that in some regular cases (see next section), the function $\frac{1}{\alpha_{1}}$ is not convex, but concave. For that case, we have the following result

Lemma 6 When $\frac{1}{\alpha_{1}}$ is concave in $\left(0,\left(L_{2}-L_{1}\right) h_{b o}\right)$, then the best-response $p_{1}^{*}\left(p_{2}\right)$ has the following characteristics: there are $-\left(L_{2}-L_{1}\right) h_{b o} \leq e_{1}$ such that 
- when $p_{2} \leq c_{1}-\left(L_{2}-L_{1}\right) h_{b o}$, then $p_{1}^{*}\left(p_{2}\right)=c_{1}$;

- when $c_{1}-\left(L_{2}-L_{1}\right) h_{b o}<p_{2} \leq c_{1}+e_{1}, p_{1}^{*}\left(p_{2}\right)=p_{2}+\left(L_{2}-L_{1}\right) h_{b o}-\epsilon$ with $\epsilon$ positive very small;

- when $c_{1}+e_{1}<p_{2}, p_{1}^{*}\left(p_{2}\right)=p_{2}$.

Thus, $p_{1}^{*}$ is continuous everywhere except at $p_{2}=c_{1}+e_{1}$, where it jumps down.

We observe that the structure of the best-response function is identical to the one identified in Lemma 5 , with $a_{1}=e_{1}$. In addition, here there is a discontinuity even when $\alpha_{1}$ is continuous.

Given the best-response functions, we are able to characterize the equilibrium of the pricing game.

Theorem 1 When $\alpha_{1}$ is convex and $\frac{1}{\alpha_{1}}$ is convex or concave in $\left(0,\left(L_{2}-L_{1}\right) h_{b o}\right)$, there exists a Nash equilibrium $\left(p_{1}^{e q}, p_{2}^{e q}\right)$ of the pricing game if and only if one of the following situations is satisfied:

1. $c_{1}-c_{2} \leq-e_{1}$, i.e., the slow supplier is so expensive that the fast supplier's best option is to price it out of the market, i.e., $p_{1}^{e q}=p_{2}^{e q}=c_{2}$.

2. $e_{2} \leq c_{1}-c_{2}$, i.e., the fast supplier is so expensive that the slow supplier's best option is to price it out of the market, i.e., $p_{1}^{e q}=c_{1}$ and $p_{2}^{e q}=c_{1}-\left(L_{2}-L_{1}\right) h_{b o}$.

3. $\lim _{\epsilon \rightarrow 0^{+}} p_{1}^{*}\left(c_{1}+e_{1}-\epsilon\right)-\left(c_{1}+e_{1}\right) \leq\left(c_{2}+e_{2}\right)-\lim _{\epsilon \rightarrow 0^{+}} p_{2}^{*}\left(c_{2}+e_{2}-\epsilon\right)$, i.e., the fast and slow suppliers can coexist and offer differentiated prices.

In any of these cases, the equilibrium is unique.

Thus, the theorem completely characterizes the types of equilibrium of the game. In any other situation, there is no equilibrium. There are three possible cases. In the first two cases, $c_{1}-c_{2} \leq-e_{1}$, or $c_{1}-c_{2} \geq e_{2}$. These are situations where the cost differential of a supplier is too large to be competitive. The third case corresponds to an equilibrium with a stable price premium of the fast supplier over the slow supplier. The technical condition guarantees that the equilibrium is indeed reached.

Interestingly, when $\frac{1}{\alpha_{1}}$ is concave, we have that $\lim _{\epsilon \rightarrow 0^{+}} p_{1}^{*}\left(c_{1}+e_{1}-\epsilon\right)-\left(c_{1}+e_{1}\right)=\left(L_{2}-L_{1}\right) h_{b o}>$ $\left(c_{2}+e_{2}\right)-\lim _{\epsilon \rightarrow 0^{+}} p_{2}^{*}\left(c_{2}+e_{2}-\epsilon\right)$, and thus the last case can never occur.

On the other hand, when $\frac{1}{\alpha_{1}}$ is convex, $\alpha_{1}$ is continuous at $\Delta=0$, and $h_{b o} \rightarrow \infty$, the last case occurs for some $c_{1}, c_{2}$. Indeed, in that case, the best-response functions are continuous, $e_{2} \rightarrow \infty, \lim _{\epsilon \rightarrow 0^{+}}\left(c_{2}+e_{2}\right)-$ $p_{2}^{*}\left(c_{2}+e_{2}-\epsilon\right) \rightarrow+\infty$, while $\lim _{\epsilon \rightarrow 0^{+}} p_{1}^{*}\left(c_{1}+e_{1}-\epsilon\right)-\left(c_{1}+e_{1}\right)$ is finite. 
Hence, we have identified two types of stable outcomes: either one supplier clearly dominates the other and captures the entire market; or both suppliers can coexist in competition, price differently and capture a portion of the market. Finally, it is also possible that no equilibrium exists. Being in one situation or another depends (1) on the cost parameters of the suppliers and the inventory costs $h_{b o}, h_{i n v}$; and (2) on the demand distribution.

\section{$6 \quad$ Exact Characterization when $L_{2}=L_{1}+1$}

In this section, we characterize $\alpha_{1}$ exactly for $L_{2}=L_{1}+1$. This will allow us to apply Theorem 1 and, in some cases, solve in closed form the price competition game between suppliers.

For this purpose, we must solve the buyer's problem, i.e., minimize the buyer's cost under all inventory policies. This is typically difficult. It involves describing the steady-state distribution of orders and inventory positions. Fortunately, when $L_{2}=L_{1}+1$, Fukuda [7] first showed that the structure of the optimal policy is tractable.

At optimality, the buyer uses two target levels $b_{1}$ and $b_{2}$ to order in the following way. At the beginning of period $t$, demand $D_{t}$ arrives. Then, if the inventory position at supplier 1, i.e., $Y_{t}^{L_{1}+1}-D_{t}$, falls below $b_{1}$, the manufacturer orders $Q_{t}^{1}=b_{1}-\left(Y_{t}^{L_{1}+1}-D_{t}\right)$ from supplier 1. Otherwise, no order is placed at supplier 1. Next, it considers the inventory position at $L_{2}$, which at this point is equal to $Y_{t}^{L_{2}}-D_{t}+Q_{t}^{1}$. If it is below $b_{2}$, it orders $Q_{t}^{2}=b_{2}-\left(Y_{t}^{L_{2}}-D_{t}+Q_{t}^{1}\right)$ from supplier 2 ; otherwise, no order is placed at supplier 2 .

\subsection{Optimal Base-Stock Levels}

The optimal policy is characterized by two base-stock levels, $b_{1}, b_{2}$, each for one supplier. Under a double base-stock policy, we have

$$
Q_{t}^{1}=\left(b_{1}-\left(Y_{t}^{L_{1}+1}-D_{t}\right)\right)^{+} \text {and } Q_{t}^{2}=\left(b_{2}-\left(Y_{t}^{L_{2}}+Q_{t}^{1}-D_{t}\right)\right)^{+} .
$$

This implies that $Q_{t}^{1}+Q_{t}^{2}=D_{t}$ always and thus $Y_{t}^{L_{2}} \equiv b_{2}$. Thus, since for $L_{1} \leq j \leq L_{2}-1$, $Y_{t}^{j}=Y_{t-1}^{j+1}-D_{t-1}+Q_{t-1}^{1}$, we establish that $Y_{t}^{j}=b_{2}+\sum_{i=1}^{L_{2}-j}\left(-D_{t-i}+Q_{t-i}^{1}\right)$. Hence,

$$
Y_{t}^{L_{1}}=b_{2}-\sum_{i=1}^{L_{2}-L_{1}} D_{t-i}+\sum_{i=1}^{L_{2}-L_{1}} Q_{t-i}^{1}
$$


In general, we do not obtain a closed-form expression for the inventory position at supplier 1, i.e. $Y_{t}^{L_{1}}$. Fortunately, this can be done when $L_{2}=L_{1}+1$. Interestingly, tractability of the problem coincides exactly with optimality of base-stock policies for two procurement modes, see Feng et al. [6]. When the two suppliers have consecutive lead times, we have that $Q_{t}^{1}=\max \left\{0, b_{1}-\left(Y_{t}^{L_{2}}-D_{t}\right)\right\}=\max \left\{0, D_{t}-\left(b_{2}-b_{1}\right)\right\}$. Thus, $Y_{t}^{L_{1}}=\max \left\{b_{2}-D_{t-1}, b_{1}\right\}$. From these two expressions, we can determine the long-run average cost for the buyer as a function of $b_{1}$ and $b_{2}$, from Equation (1). It is easy to see that if both suppliers are used, i.e., $b_{1}<b_{2}$, then

$$
\overline{Q^{1}}=\int_{b_{2}-b_{1}}^{\infty} \bar{F}(q) d q \text { and } \overline{Q^{2}}=\int_{0}^{b_{2}-b_{1}} \bar{F}(q) d q
$$

Also, with a recursion similar as the one above, we have that $Y_{t}^{0}=Y_{t-L_{1}}^{L_{1}}-\sum_{i=1}^{L_{1}} D_{t-i}$. $Y_{0}$ is thus simply $Y^{L_{1}}$ minus the sum of $L_{1}$ demand realizations, where the stationary distribution of $Y^{L_{1}}$ is known. Indeed, by construction, $Y^{L_{1}}=\max \left\{b_{2}-D, b_{1}\right\}$ and hence

$$
\begin{aligned}
& \mathbb{P}\left(Y^{L_{1}}=b_{1}\right)=\bar{F}\left(b_{2}-b_{1}\right), \text { and } \\
& \mathbb{P}\left(Y^{L_{1}} \leq q\right)=\bar{F}\left(b_{2}-q\right), \text { for } q \geq b_{1} .
\end{aligned}
$$

By convoluting this distribution with the total demand during $L_{1}$ periods, we can compute $\mathbb{E} h\left(Y^{0}\right)$ as a function of $b_{1}, b_{2}$. When $L_{1}>0$,

$$
\begin{aligned}
\mathbb{E} h\left(Y^{0}\right)= & h_{\text {inv }}\left(\bar{F}\left(b_{2}-b_{1}\right) \int_{u=0}^{b_{1}}\left(b_{1}-u\right) f_{L_{1}}(u) d u+\int_{v=0}^{b_{2}-b_{1}} \int_{u=0}^{b_{2}-v}\left(b_{2}-v-u\right) f(v) f_{L_{1}}(u) d u d v\right) \\
+ & h_{b o}\left(\bar{F}\left(b_{2}-b_{1}\right) \int_{u=b_{1}}^{\infty}\left(u-b_{1}\right) f_{L_{1}}(u) d u+\int_{v=0}^{b_{2}-b_{1}} \int_{u=b_{2}-v}^{\infty}\left(u+v-b_{2}\right) f(v) f_{L_{1}}(u) d u d v\right)
\end{aligned}
$$

To select the optimal base-stock policy, we set $\frac{d C}{d b_{1}}=\frac{d C}{d b_{2}}=0$. The first condition yields

$$
\frac{d C}{d b_{1}}=\Delta \bar{F}\left(b_{2}-b_{1}\right)+h_{i n v} \bar{F}\left(b_{2}-b_{1}\right)\left(1-\bar{F}_{L_{1}}\left(b_{1}\right)\right)-h_{b o} \bar{F}\left(b_{2}-b_{1}\right) \bar{F}_{L_{1}}\left(b_{1}\right)=0
$$

or equivalently,

$$
\bar{F}_{L_{1}}\left(b_{1}\right)=\frac{h_{i n v}+\Delta}{h_{i n v}+h_{b o}}
$$

Notice that this implies that, when $\Delta \geq h_{b o}$, it is best not to order anything with the fast supplier, i.e., $b_{1}=-\infty$, as we already knew. The second condition is 


$$
\begin{aligned}
\frac{d C}{d b_{2}} & =-\Delta \bar{F}\left(b_{2}-b_{1}\right)+h_{i n v} \int_{v=0}^{b_{2}-b_{1}} f(v)\left(1-\bar{F}_{L_{1}}\left(b_{2}-v\right)\right) d v-h_{b o} \int_{v=0}^{b_{2}-b_{1}} f(v) \bar{F}_{L_{1}}\left(b_{2}-v\right) d v \\
& =h_{i n v}-\left(\Delta+h_{i n v}\right) \bar{F}\left(b_{2}-b_{1}\right)-\left(h_{i n v}+h_{b o}\right) \int_{v=0}^{b_{2}-b_{1}} f(v) \bar{F}_{L_{1}}\left(b_{2}-v\right) d v \\
& =h_{i n v}-\left(h_{i n v}+h_{b o}\right)\left(\bar{F}\left(b_{2}-b_{1}\right) \bar{F}_{L_{1}}\left(b_{1}\right)+\int_{v=0}^{b_{2}-b_{1}} f(v) \bar{F}_{L_{1}}\left(b_{2}-v\right) d v\right) \\
& =0
\end{aligned}
$$

or in other words

$$
\int_{v=0}^{\infty} f(v) \bar{F}_{L_{1}}\left(\max \left\{b_{1}, b_{2}-v\right\}\right) d v=\frac{h_{i n v}}{h_{\text {inv }}+h_{b o}} .
$$

This, plugging in $b_{1}$ from Equation (6), yields the optimal $b_{2}$.

When $L_{1}=0$, a straightforward analysis yields that the optimal base-stock for the fast supplier is $b_{1}=0$, i.e., order for immediate delivery if and only if there is backlog, which is intuitive. In addition, Equation (7) is replaced by

$$
\begin{array}{ll}
\bar{F}\left(b_{2}\right)=\frac{h_{i n v}}{h_{i n v}+\Delta} & \text { when } 0 \leq \Delta \leq h_{b o}, \\
\bar{F}\left(b_{2}\right)=\frac{h_{i n v}}{h_{i n v}+h_{b o}} & \text { otherwise. }
\end{array}
$$

\subsection{Equilibrium Analysis with Immediate Deliveries}

In what follows, we consider the specific case $L_{1}=0$ and $L_{2}=1$. This corresponds to the most extreme situation, where the fast supplier allows immediate delivery, i.e., with no demand risk. Although the same results can be obtained for $L_{1}>0$, we focus on this simpler case, since the insights obtained are representative of the general case.

As mentioned above, in this case, the base-stock level for the fast supplier is $b_{1}=0$ when $\Delta \leq h_{b o}$, and $b_{1}=-\infty$ otherwise. Through Equation (8), we see that when the difference $\Delta$ goes to zero, $b_{2}$ goes to the lower bound of the demand, $D_{\min }$. Thus $\alpha_{2} \rightarrow \frac{D_{\min }}{\bar{D}}$. Note, however, that when $\Delta=0, \alpha_{2}=0$, that is, the market share function $\alpha_{1}$ may be discontinuous at $\Delta=0$, depending on whether $D_{\min }=0$ or not. It is also discontinuous at $\Delta=h_{b o}$. Otherwise, when $0<\Delta<h_{b o}, \alpha_{1}=\frac{1}{\bar{D}} \int_{b_{2}}^{\infty} \bar{F}(q) d q$, or, equivalently after the change of variables $v=\bar{F}(q)$,

$$
\alpha_{1}=\frac{1}{\bar{D}} \int_{0}^{\frac{h_{i n v}}{h_{i n v}+\Delta}} \frac{v d v}{f\left(\bar{F}^{-1}(v)\right)} .
$$

We can show the following result. 
Lemma 7 If the demand has a non-decreasing failure rate, i.e., $\frac{f}{\bar{F}}$ is non-decreasing, then the market share function $\alpha_{1}$ is convex in $\Delta \in\left(0, h_{b o}\right)$.

This lemma, together with Lemma 2, implies that when the demand failure rate is non-decreasing, then the profit function of the slow supplier, 2, is well-behaved.

Similarly, in order to use Lemma 4, we would like to establish that $\frac{1}{\alpha_{1}}$ is convex. Unfortunately, the condition turns out not to be trivial. Thus, the profit function of the fast supplier, 1, may not behave nicely. The following examples serve as illustrations.

Example 1 When the demand is uniform in $\left[D_{\min }, D_{\max }\right]$, then $\alpha_{1}=\frac{D_{\max }-D_{\min }}{2 \bar{D}}\left(\frac{h_{i n v}}{h_{i n v}+\Delta}\right)^{2}$. Thus, $\alpha_{1}$ and $\frac{1}{\alpha_{1}}$ are convex in $\left(0, h_{b o}\right)$.

Example 2 When the demand is exponential with rate $\lambda$, then $\alpha_{1}=\frac{h_{i n v}}{h_{i n v}+\Delta}$. Thus, $\alpha_{1}$ and $\frac{1}{\alpha_{1}}$ are convex in $\left(0, h_{b o}\right)$.

Example 3 When the demand is Pareto with rate $\beta \geq 1$, i.e., $\bar{F}(q)=(1+q)^{-\beta}$, then $\alpha_{1}=\left(\frac{h_{\text {inv }}}{h_{\text {inv }}+\Delta}\right)^{1-1 / \beta}$. Thus, $\alpha_{1}$ is convex and $\frac{1}{\alpha_{1}}$ is concave in $\left(0, h_{b o}\right)$.

Example 4 When the demand is normal with mean $\mu$ and standard deviation $\sigma$, then $\alpha_{1}$ is convex and $\frac{1}{\alpha_{1}}$ is concave in $\left(0, h_{b o}\right)$.

Hence, in all four example, we can use Lemmas 3,5 or 6. Below, we characterize the equilibrium explicitly for the uniform and exponential distributions, for $h_{b o}=\infty$.

Theorem 2 Assume that the demand is uniform in $\left[D_{\min }, D_{\max }\right]$. Then,

- when

$$
\frac{c_{1}-c_{2}}{h_{\text {inv }}} \leq-\left(\frac{1}{2}-\frac{1}{2} \sqrt{1-\frac{D_{\max }-D_{\min }}{D_{\max }+D_{\min }}}\right) \leq 0,
$$

then there is a unique equilibrium $p_{1}^{e q}=p_{2}^{e q}=c_{2}$;

- when

$$
\frac{c_{1}-c_{2}}{h_{i n v}} \geq \frac{\left(1+\sqrt{1-\frac{D_{\max }-D_{\min }}{D_{\max }+D_{\min }}}\right)^{3}}{2\left(\frac{D_{\max }-D_{\min }}{D_{\max }+D_{\min }}\right)}-1
$$


then there is a unique equilibrium with

$$
\frac{\Delta}{h_{i n v}}=\left\{2\left(\frac{D_{\max }-D_{\min }}{D_{\max }+D_{\min }}\right)\left(1+\frac{c_{1}-c_{2}}{h_{\text {inv }}}\right)\right\}^{1 / 3}-1
$$

- otherwise, there is no equilibrium.

The theorem illustrates that even in the simplest case, for the uniform distribution, we find situations where there is no equilibrium. In addition, the theorem provides some insight on the role of the variability of the distribution on the equilibria.

Namely, given $D_{\max }$, when $D_{\min }=0$, the uniform distribution has the largest spread. In that case, we see that the third case never occurs, as the two first cases cover every possibility: there is always an equilibrium. The intuition is that variability reduces the incentive of the fast supplier to outprice the slow supplier, since the "prize" of capturing the entire market by setting $\Delta=0$, compared to the share of settling for $\Delta=\epsilon$ very small, is zero. Thus, the fast supplier's strategy is more stable (i.e., its best-response function is continuous) and hence the equilibrium with $\Delta>0$ can be reached.

When $D_{\min } \rightarrow D_{\max }$, on the other hand, the market share difference between $\Delta=0$ and $\Delta=\epsilon$ very small is huge. Competition is thus very much focused on outbidding the competitor. Thus, we do not have an equilibrium when $\frac{c_{1}-c_{2}}{h_{i n v}}>0$.

Interestingly, when $c_{1}=c_{2}$, then there is equilibrium if and only if $\frac{D_{\max }-D_{\min }}{D_{\max }+D_{\min }} \geq-8+4 \sqrt{5} \approx 0.944$. Again, the larger the variability, the smaller the set of costs where equilibrium exists.

Figure 4 illustrates the three cases of the theorem, for a uniform distribution in $[1,2]$.

We present below a similar result for the exponential distribution.

Theorem 3 Assume that the demand is exponential. Then there is always a unique Nash equilibrium:

- when $\frac{c_{1}-c_{2}}{h_{\text {inv }}} \leq-1$, then there is a unique equilibrium $p_{1}^{e q}=p_{2}^{e q}=c_{2}$;

- otherwise, at equilibrium

$$
\frac{\Delta}{h_{i n v}}=-\frac{1}{2}+\sqrt{\frac{c_{1}-c_{2}}{h_{i n v}}+\frac{5}{4}}
$$

In this case, we observe that there is always an equilibrium. Note that when $c_{1}=c_{2}$, the equilibrium is such that $\Delta \approx 0.62 h_{i n v}>0$. Thus, it is optimal for the fast supplier not to compete solely in price, trying to outbid the slow supplier, but rather to charge a premium and share the market with the slow supplier. 


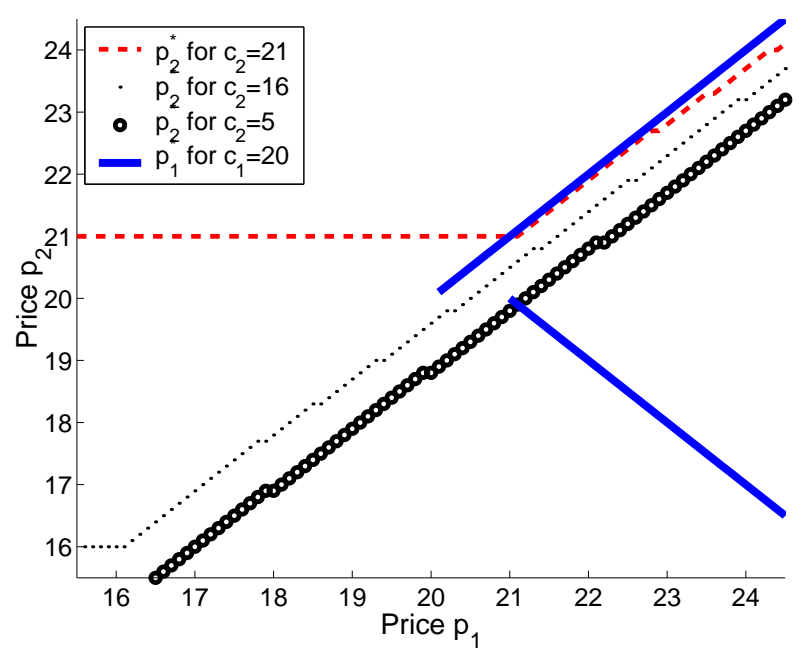

Figure 4: Best response functions $p_{1}^{*}\left(p_{2}\right)$ and $p_{2}^{*}\left(p_{1}\right)$ for a uniform demand $[1,2], h_{i n v}=1$ and a cost $c_{1}=20$. We see that, depending on the value of $c_{2}$, we may or may not have an equilibrium. We illustrate the three cases of the theorem: for $c_{2}=21$, then at equilibrium $\Delta=0$ and $p_{1}^{e q}=p_{2}^{e q}=c_{2}=21$; when $c_{2}=16$, then we fall into the no-equilibrium case; and when $c_{2}=5, \frac{c_{1}-c_{2}}{h_{i n v}}$ is large enough, and at equilibrium $\Delta>0$.

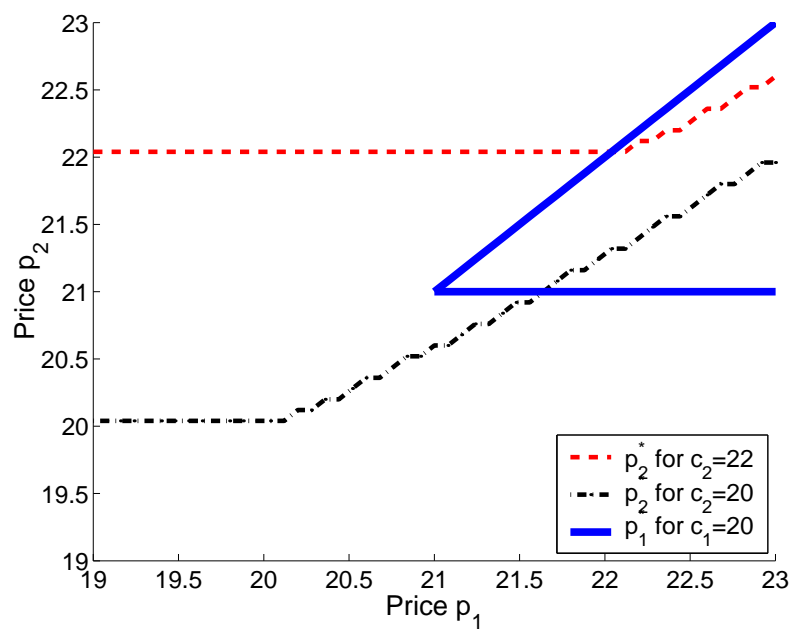

Figure 5: Best response functions $p_{1}^{*}\left(p_{2}\right)$ and $p_{2}^{*}\left(p_{1}\right)$ for an exponential demand, $h_{i n v}=1$ and a cost $c_{1}=20$. We see that, depending on the value of $c_{2}$, we have an equilibrium with $\Delta=0$ (when $c_{2}=22$ ), or with $\Delta>0\left(\right.$ when $\left.c_{2}=c_{1}=20\right)$. 
For the uniform and exponential distributions, when stocking out is very expensive, i.e., $h_{b o}=\infty$, we have found three possible competitive situations, already identified in Theorem 1. First, when the fast supplier is significantly cheaper than the slow supplier, it will undercut its price and capture all the market, in the same way as in the Bertrand competition model. Second, when the cost difference is medium, there may be no equilibrium. And third, when the price difference is low (could be positive as for the exponential distribution, or negative as for the uniform), the suppliers stabilize in a situation where prices are differentiated, i.e., $\Delta>0$, and share the market. It is also interesting that $\Delta$ grows with $c_{1}-c_{2}$, but that it grows less than linearly.

\section{Approximation of Equilibria}

As we have seen, the market share function $\alpha_{1}$ determines completely whether the pricing game has an equilibrium. In the previous section, we were able to characterize $\alpha_{1}$ explicitly, because the inventory problem is tractable for $L_{2}=L_{1}+1$. In this section, we develop an approximation for the general case, for $L_{2}>L_{1}+1$. First, we assume that the buyer uses a double base-stock inventory policy. Second, we develop the approximation for $\Delta \approx 0$.

As we have seen, $\alpha_{1}$ is very steep for small values of $\Delta$ : this plays a critical role in the game, as the steepness represents greater importance of price over the lead time difference. Thus, it determines the incentive of the fast supplier to outbid the slow supplier with a lower price. For this reason, we choose to develop an approximation of $\alpha_{1}$ around $\Delta=0$.

In addition, computationally, $\alpha_{1}$ is difficult to calculate for small $\Delta$, since the optimal base-stock levels for the buyer are very sensitive to $\Delta$. To find numerically these optimal levels takes a very long time. Moreover, since optimization requires simulation, to obtain a higher accuracy, the size of the simulation grows enormously. The asymptotic approximation of the market shares around $\Delta=0$ can thus also be used to alleviate computational effort.

We start by observing that, when $\Delta>0$ and very small, the slow supplier will receive an order for $D_{\min }$ every period, and the fast supplier an order for the rest. Thus

$$
\lim _{\Delta \rightarrow 0^{+}} \alpha_{1}(\Delta)=1-\frac{D_{\min }}{\bar{D}} .
$$

At this point, we are interested in approximating $\left(1-\frac{D_{\min }}{\bar{D}}\right)-\alpha_{1}(\Delta)$ for small $\Delta$. For this purpose, we conducted an extensive simulation for different lead-time parameters, and $h_{i n v}=1, h_{b o}=9$. For each 
case, we simulated 50000 period of a uniform demand $[0,2]$ (and hence $D_{\min }=0, \bar{D}$ ), and we computed the average cost of double base-stock policies. Among these, we selected the one that minimized the buyer's average cost, and this for different $\Delta \in[0,0.1]$. We hence calculated $\alpha_{1}$ for different $\Delta$. We found that $1-\alpha_{1}(\Delta)$ varied with $\Delta^{b}$. Figure 6 shows the variation of $\log \left(1-\alpha_{1}\right)$ with $\log (\Delta)$.
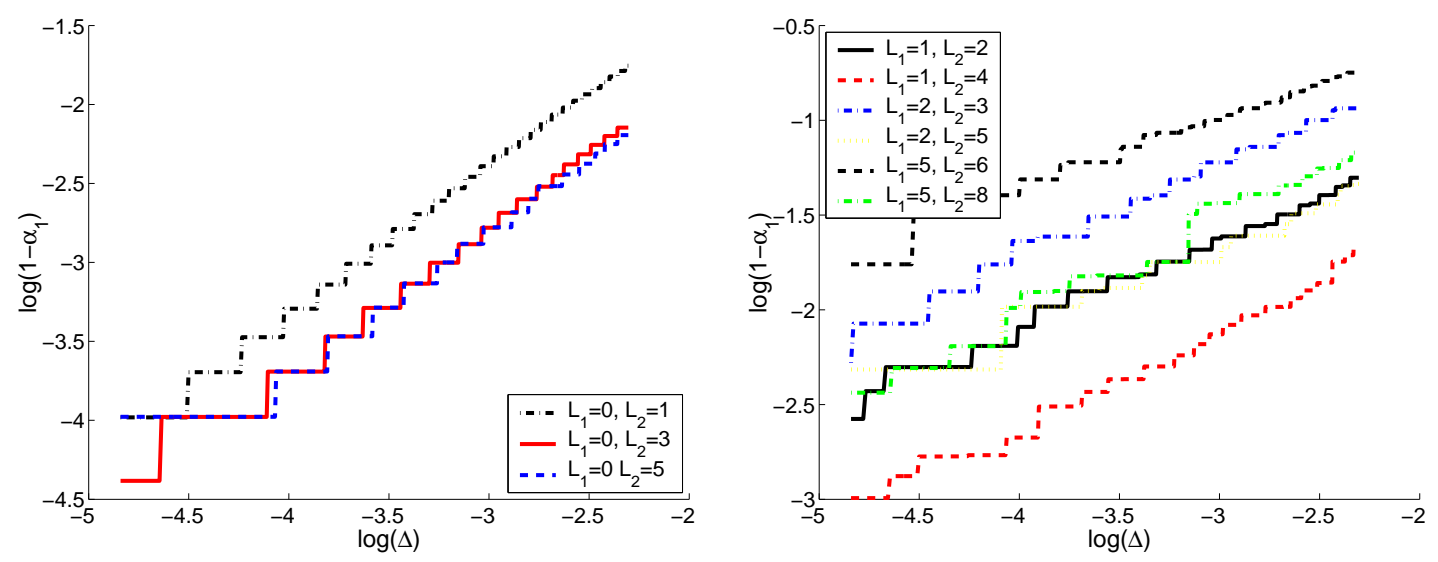

Figure 6: Plot of $\log \left(\left(1-\frac{D_{\min }}{\bar{D}}\right)-\alpha_{1}(\Delta)\right)$ as a function of $\log (\Delta)$, for various lead-time parameters. Clearly, we see that the behavior for the cases where $L_{1}=0$ (left) is different to the rest $\left(L_{1}>0\right.$, right), as the slopes are around 1 (left) and around 0.5 (right).

Since the relationship was roughly linear, we calculated the slope and intercept of the regression $\log (1-$ $\left.\alpha_{1}\right)=S L O P E \cdot \log (\Delta)+I N T E R C E P T$. This is summarized in Figure 7.

The simulation results seem to imply that two types of behaviors can occur:

- Either $\left(1-\frac{D_{\min }}{\bar{D}}\right)-\alpha_{1}(\Delta)$ grows linearly with $\Delta$. This corresponds in the simulation to the case $L_{1}=0$.

- Or $\left(1-\frac{D_{\min }}{\bar{D}}\right)-\alpha_{1}(\Delta)$ grows with the square-root of $\Delta$. This appeared in all the cases with $L_{1}>0$.

We have performed similar simulations for a truncated normal distribution with $\mu=1$ and $\sigma=0.3$, and we have found similar different behavior between the cases $L_{1}=0$ and $L_{1}>0$.

To explain these two types of behavior, we develop an approximation of the market share function that indeed identifies these two situations. The approximation is detailed in the appendix, but the main steps 


\begin{tabular}{|c|c|c|c|}
$L_{1}$ & $L_{2}$ & slope & intercept \\
\hline 0 & 1 & 0.9436 & 0.4371 \\
0 & 3 & 0.9227 & -0.0083 \\
0 & 5 & 0.8668 & -0.2079 \\
\hline 1 & 2 & 0.4560 & -0.2710 \\
1 & 4 & 0.5239 & -0.5431 \\
1 & 6 & 0.4239 & -0.9188 \\
2 & 3 & 0.4829 & 0.2097 \\
2 & 5 & 0.4228 & -0.4020 \\
2 & 7 & 0.5640 & -0.1791 \\
5 & 6 & 0.3743 & 0.1310 \\
5 & 8 & 0.5186 & 0.0374 \\
5 & 10 & 0.4738 & -0.1819 \\
\hline
\end{tabular}

Figure 7: Summary of regression coefficients of $\log \left(1-\alpha_{1}\right)=S L O P E \cdot \log (\Delta)+I N T E R C E P T$, for each of the simulation cases.

are described next. First, we approximate the cost of the buyer as

$$
\begin{aligned}
\tilde{C}= & p_{2} \bar{D}+\mathbb{P}\left(D_{t}>\frac{b_{2}-b_{1}}{L_{2}-L_{1}}\right) \mathbb{E}_{D_{t}>\frac{b_{2}-b_{1}}{L_{2}-L_{1}}}\left\{\begin{array}{c}
\Delta\left(D_{t}-\frac{b_{2}-b_{1}}{L_{2}-L_{1}}\right) \\
+h_{i n v}\left(b_{1}-D_{t+1}-\ldots-D_{t+L_{1}}\right)^{+} \\
-h_{b o}\left(b_{1}-D_{t+1}-\ldots-D_{t+L_{1}}\right)^{-}
\end{array}\right\} \\
+ & \mathbb{P}\left(D_{t} \leq \frac{b_{2}-b_{1}}{L_{2}-L_{1}}\right) \mathbb{E}_{D_{t} \leq \frac{b_{2}-b_{1}}{L_{2}-L_{1}}}\left\{\begin{array}{c}
+h_{i n v}\left(b_{1}+\frac{b_{2}-b_{1}}{L_{2}-L_{1}}-D_{t}-D_{t+1}-\ldots-D_{t+L_{1}}\right)^{+} \\
-h_{b o}\left(b_{1}+\frac{b_{2}-b_{1}}{L_{2}-L_{1}}-D_{t}-D_{t+1}-\ldots-D_{t+L_{1}}\right)^{-}
\end{array}\right\}
\end{aligned}
$$

As a result, the optimal value for $b_{2}-b_{1}$ satisfies

$$
\Delta=\left(h_{i n v}+h_{b o}\right) \mathbb{P}\left(b_{1} \leq D_{t+1}+\ldots+D_{t+L_{1}} \leq b_{1}+\frac{b_{2}-b_{1}}{L_{2}-L_{1}}-D_{t}\right) .
$$

In addition, we have that $1-\alpha_{1}(\Delta)=\frac{1}{\bar{D}} \frac{b_{2}-b_{1}}{L_{2}-L_{1}}$. Equation (10 yields two cases with significantly different behavior.

- If $L_{1}=0$, then Equation (10) can be rewritten as

$$
\frac{\Delta}{h_{\text {inv }}+h_{b o}}=\mathbb{P}\left(\frac{b_{2}-b_{1}}{L_{2}-L_{1}} \geq D_{t}\right)
$$

which implies that $1-\alpha_{1}(\Delta)=\frac{1}{\bar{D}} F^{-1}\left(\frac{\Delta}{h_{i n v}+h_{b o}}\right)$. 
Thus, when $f\left(D_{\text {min }}\right)>0, F(q) \approx f\left(D_{\text {min }}\right)\left(q-D_{\text {min }}\right)$, which implies that $1-\alpha_{1}(\Delta) \approx \frac{D_{\text {min }}}{\bar{D}}+$ $\frac{1}{f\left(D_{\min }\right) \bar{D}}\left(\frac{\Delta}{h_{\text {inv }}+h_{b o}}\right)$. We this observe a linear variation of the market share for small $\Delta$, just what we found for $L_{1}=0$ in Figure 6. Hence,

$$
\alpha_{1}(\Delta)=\left(1-\frac{D_{\min }}{\bar{D}}\right)-k\left(\frac{\Delta}{h_{i n v}+h_{b o}}\right)
$$

Note, however, that when $f\left(D_{\min }\right)=0$, the variation is no longer linear. When $F(q) \approx a\left(q-D_{\min }\right)^{b}$, with $b>1$ (in which case $f\left(D_{\min }\right)=0$ ), the approximation becomes

$$
\alpha_{1}(\Delta)=\left(1-\frac{D_{\min }}{\bar{D}}\right)-k\left(\frac{\Delta}{h_{i n v}+h_{b o}}\right)^{1 / b}
$$

This situation takes place for distributions such as a truncated normal.

- If $L_{1}>0$, then Equation (10) can be approximated by

$$
\frac{\Delta}{h_{\text {inv }}+h_{b o}} \approx f_{L_{1}}\left(b_{1}\right) \mathbb{E}\left(\frac{b_{2}-b_{1}}{L_{2}-L_{1}}-D_{t}\right)^{+}
$$

Again, when $f\left(D_{\text {min }}\right)>0, \mathbb{E}\left(q-D_{t}\right)^{+} \approx \frac{1}{2} f\left(D_{\text {min }}\right)\left(q-D_{\text {min }}\right)^{2}$ and hence

$$
\frac{\Delta}{h_{i n v}+h_{b o}} \approx \frac{1}{2} f_{L_{1}}\left(b_{1}\right) f\left(D_{\min }\right)\left(\frac{b_{2}-b_{1}}{L_{2}-L_{1}}-D_{\min }\right)^{2} .
$$

and hence $1-\alpha_{1}(\Delta) \approx \frac{D_{\min }}{\bar{D}}+\frac{1}{\bar{D}} \sqrt{\frac{2}{f_{L_{1}}\left(b_{1}\right) f\left(D_{\min }\right)}} \sqrt{\frac{\Delta}{h_{\text {inv }}+h_{b o}}}$. This implies that the market share for small $\Delta$ decreases with the square root of $\Delta$, as noticed in Figure 6. Hence,

$$
\alpha_{1}(\Delta)=\left(1-\frac{D_{\min }}{\bar{D}}\right)-k \sqrt{\frac{\Delta}{h_{i n v}+h_{b o}}} .
$$

Finally, when $f\left(D_{\text {min }}\right)=0$, and $F(q) \approx a\left(q-D_{\text {min }}\right)^{b}$, with $b>1$, then $\mathbb{E}\left(q-D_{t}\right)^{+} \approx \frac{a}{b+1}\left(q-D_{\text {min }}\right)^{b+1}$ and hence

$$
\alpha_{1}(\Delta)=\left(1-\frac{D_{\min }}{\bar{D}}\right)-k\left(\frac{\Delta}{h_{i n v}+h_{b o}}\right)^{1 /(b+1)}
$$

Thus, we see that the market share of the fast supplier, 1, typically decreases either linearly with $\Delta$, when $L_{1}=0$, or with the square-root of $\Delta$, when $L_{1}>0$, for small price differences, as described by Equations (11) and (13).

It is interesting to see that the effect of inventory cost, $h_{i n v}+h_{b o}$, only plays a role as a scaling factor of $\Delta$. In addition, the lead time difference does not play a role in the approximation, only $L_{1}$ does. 
In what follows, we compute approximate equilibria, for the case $L_{1}>0, D_{\min }=0$. Keeping in mind that the market share must be positive, the analysis is done for $0 \leq \Delta \leq \frac{h_{i n v}+h_{b o}}{k^{2}}$. Within this range, we have that $\alpha_{1}$ is convex, but it turns out that $\frac{1}{\alpha_{1}}$ is neither convex or concave. Thus, we cannot use Theorem 1. However, we can easily derive, for this particular market share function, the equilibrium outcomes.

Theorem 4 When

$$
\alpha_{1}(\Delta)=\left(1-k \sqrt{\frac{\Delta}{h_{i n v}+h_{b o}}}\right)^{+}
$$

then,

- when

$$
\frac{c_{1}-c_{2}}{h_{i n v}+h_{b o}} \leq-\frac{1}{4 k^{2}}
$$

then there is a unique equilibrium $p_{1}^{e q}=p_{2}^{e q}=c_{2}$, and hence $\alpha_{1}\left(\Delta^{e q}\right)=1$;

- when

$$
-\frac{1}{4 k^{2}} \leq \frac{c_{1}-c_{2}}{h_{i n v}+h_{b o}} \leq \frac{1}{4 k^{2}}
$$

then there is no equilibrium;

- when

$$
\frac{1}{4 k^{2}} \leq \frac{c_{1}-c_{2}}{h_{i n v}+h_{b o}} \leq \frac{3}{k^{2}}
$$

then there is a unique equilibrium with

$$
\frac{\Delta}{h_{i n v}+h_{b o}}=\left(\frac{1}{5 k^{2}}+\sqrt{\frac{1}{5} \frac{c_{1}-c_{2}}{h_{i n v}+h_{b o}}+\frac{1}{25 k^{2}}}\right)^{2}>0 ;
$$

- otherwise, when

$$
\frac{c_{1}-c_{2}}{h_{i n v}+h_{b o}} \geq \frac{3}{k^{2}}
$$

then there is a unique equilibrium $p_{1}^{e q}=c_{1}$ and $p_{2}^{e q}=c_{1}-\frac{h_{i n v}+h_{b o}}{k^{2}}$, and hence $\alpha_{1}\left(\Delta^{e q}\right)=0$.

The theorem identifies the same type of equilibria obtained in Theorem 1. Again, when there is an equilibrium, either one of the suppliers is wiped out the market $\left(c_{1}-c_{2}\right.$ very large or very small), or both coexist with a positive price difference. That only occurs when $\frac{1}{4 k^{2}} \leq \frac{c_{1}-c_{2}}{h_{i n v}+h_{b o}} \leq \frac{3}{k^{2}}$. 


\section{Conclusion}

As we have seen, including lead time considerations into price competition leads to significantly more complex results than the ones predicted by the traditional asymmetric Bertrand model. We have identified three different equilibrium types, both in the exact and the approximated models. Depending on the range of costs, and the type of demand distribution, we obtain one sort of equilibrium or another.

(A) When $c_{1}-c_{2}$ is very small (negative), supplier 1 takes supplier 2 out of the market. The equilibrium price is $p_{1}^{e q}=c_{2}$, i.e., the fast supplier prices low enough to put supplier 2 out of business. This is identical to the standard asymmetric Bertrand result.

(B) When $c^{1}-c^{2}$ is medium low, no equilibrium exists. We have observed the result both for the exact model for the uniform distribution; exact with demand such that $1 / \alpha_{1}$ is concave, e.g., the normal demand; and the approximated model.

(C) When $c^{1}-c^{2}$ is medium high, suppliers share the orders of the buyer, while offering different prices. This situation did not appear in the asymmetric Bertrand result. We have identified a case where price differentiation is stable, and a higher cost supplier with a lead time advantage can capture some business, offering more expensive express delivery.

(D) When $c^{1}-c^{2}$ is very high, the fast supplier cannot offer a low enough price to be competitive with the slow supplier. As a result, $p_{2}^{e q}$ is set sufficiently low to ensure that by offering $p_{1}^{e q}=c_{1}$, the fast supplier does not capture any market share.

One of the questions raised by our results is to understand how to deal with case (B): no equilibrium. There, the pricing game between the suppliers does not have a set of static pure strategies, that are unilaterally optimal for the players. The best-response functions to a competitor's price are well-defined, but do not lead to an equilibrium, as they are discontinuous. In reality, in a dynamic setting, one may see suppliers moving prices up and down, reacting to each other's prices, and never converging to a stable static price. Thus, one could analyze the dynamic pricing game, and derive subgame-perfect equilibria. However, this is technically very challenging, since then the buyer behaves strategically, possibly adopting dynamic base-stock policies as well. Another option is to consider mixed strategies; this is not appropriate in our game since we are considering a one-shot game in prices in an infinite-horizon replenishment setting and in such an infinite horizon, the player would observe their competitor's price and adjust theirs immediately. 
Hopefully, the other types of equilibria found in the analysis are consistent with academic research as well as practice. Cases (A) and (D) are examples of deterrence pricing: the most competitive supplier sets a price so that its competitor cannot profitably enter the market. Case $(\mathrm{C})$ shows that it is stable to have the fast and expensive supplier charges a premium for fast delivery. In essence, this fast supplier gives up on competing on low cost and settles for a more reasonable strategy of higher margin and smaller volume. This type of strategies can be observed in the Adidas and Trigema mentioned in the introduction.

At this point, we would like to point out several extensions to the existing results. First of all, the infinite-horizon average profit analysis can be extended to include the discounted profit case. Also, when such a long horizon is not consistent with reality, e.g., for short life-cycle products, sourcing with suppliers with different lead times may be an even more interesting strategy for the buyer, since there is a limited potential to serve demand with the longer lead time supplier. We hence expect, for the same cost difference, to observe larger price differences in equilibrium.

Finally, in our model, we have assumed that there was no fixed cost associated with an order. This naturally lead to using base-stock policies with each supplier. What happens when there are set-up costs? The buyer would purchase components using an $(s, S)$ policy with each supplier. The pricing game could be studied in this situation, where the fixed charge $K_{i}$, the variable cost $c_{i}$ and the lead time $L_{i}$ are given inputs, and the price $p_{i}$ is the strategy of the supplier.

\section{References}

[1] Allon G. and A. Federgruen 2003. "Competition in Service Industries." Working Paper, Columbia University.

[2] Anupindi R. and R. Akella 1993. "Diversification under Supply Uncertainty." Management Science, 39(8), pp. 944-963.

[3] Babich V., A. N. Burnetas and P. H. Ritchken 2003. "Competition and Diversification Effects in Supply Chains with Supplier Default Risk." Working Paper, Industrial and Operations Engineering Department, University of Michigan.

[4] Beil D. and L. M. Wein 2002. "An Inverse-Optimization-Based Auction Mechanism to Support a Multiattribute RFQ Process." Management Science, 49(11), pp. 1529-1545. 
[5] Federgruen A. and N. Yang 2005. "Safeguarding Strategic Supplies: Planning for Disaster." Working paper, Graduate School Of Business, Columbia University.

[6] Feng Q., G. Gallego, S. P. Sethi, H. Yan and H. Zhang 2004. "Optimality and Nonoptimality of the Base-stock Policy in Inventory Problems with Multiple Delivery Modes.” Working paper.

[7] Fukuda Y. 1964. "Optimal Policies for the Inventory Problem with Negotiable Leadtime." Management Science, 10(4), pp. 690-708.

[8] Gerchak Y. and M. Parlar 1990. "Yield Randomness / Cost Tradeoffs and Diversification in the EOQ Model" Naval Research Logistics, 37(3), pp. 341-354.

[9] Grupp W., Trigema CEO. Panel Discussion, WirtschaftsWoche Konferenz: Beste Fabrik, Nürnberg, October 25-26, 2004.

[10] Lariviere M. A. and E. L. Porteus 2001. "Selling to the Newsvendor: An Analysis of Price-Only Contracts." Manufacturing $\&$ Service Operations Management, 3(4), pp. 293-305.

[11] Martínez-de-Albéniz V. 2004. "Portfolio Strategies in Supply Contracts." Ph.D. dissertation, OR Center, MIT.

[12] Minner S. 2003. "Multiple-supplier Inventory Models In Supply Chain Management: a Review." Int. J. Production Economics, 81-82, pp. 265-279.

[13] Moinzadeh K. and S. Nahmias 1988. "A Continuous Review Model for an Inventory System with Two Supply Modes." Management Science, 34(6), pp. 761-773.

[14] Moinzadeh K. and C. P. Schmidt 1991. "An $(S-1, S)$ Inventory System With Emergency Orders." Operations Research, 32(2), pp. 308-321.

[15] Parlar M. and D. Wang 1993. "Diversification Under Yield Randomness in Inventory Models." European Journal of Operational Research, 66(1), pp. 52-64.

[16] Tagaras G. and D. Vlachos 2001. "A Periodic Review Inventory System With Emergency Replenishments." Management Science, 47(3), pp. 415-429.

[17] Tirole J. 1988. The Theory of Industrial Organization. MIT Press, Cambridge, Massachusetts. 
[18] Tomlin B. 2006. "On the Value of Mitigation and Contingency Strategies for Managing Supply Chain Disruption Risks." Management Science, 52(5), pp. 639-657.

[19] Vives X. 1999. Oligopoly Pricing. MIT Press, Cambridge, Massachusetts.

[20] Whittemore A. S. and S. C. Saunders 1977. "Optimal Inventory Under Stochastic Demand With Two Supply Options." SIAM Journal of Applied Mathematics, 32(2), pp. 293-305. 


\section{A Proofs}

\section{A.1 Proof of Lemma 2}

Proof. If $\alpha_{1}$ is convex then $\frac{d^{2} \alpha_{1}}{d \Delta^{2}} \geq 0$. Let $p_{2} \in\left(p_{1}-\left(L_{2}-L_{1}\right) h_{b o}, p_{1}\right)$ satisfy the first-order condition, i.e.,

$$
\frac{d \Pi_{2}}{d p_{2}}=-\left(p_{2}-c_{2}\right) \frac{d \alpha_{2}}{d \Delta}+\alpha_{2}=\left(p_{2}-c_{2}\right) \frac{d \alpha_{1}}{d \Delta}+1-\alpha_{1}=0 .
$$

Then,

$$
\frac{d^{2} \Pi_{2}}{d p_{2}^{2}}=-\left(p_{2}-c_{2}\right) \frac{d^{2} \alpha_{1}}{d \Delta^{2}}+2 \frac{d \alpha_{1}}{d \Delta} \leq 0
$$

Thus $\Pi_{2}$ must be increasing and then decreasing, and hence pseudo-concave.

\section{A.2 Proof of Lemma 3}

Proof. When $\alpha_{1}$ is convex, using Lemma 2 , we have that $\Pi_{2}\left(p_{1}, p_{2}\right)$ has a unique maximum in $\left(p_{1}-\left(L_{2}-\right.\right.$ $\left.\left.L_{1}\right) h_{b o}, p_{1}\right)$. Let $p_{2}^{0}$ be this maximizer, and let $p_{2}^{0}=p_{1}$ when the maximum is achieved when $p_{2} \rightarrow p_{1}$, and $p_{2}^{0}=p_{1}-\left(L_{2}-L_{1}\right) h_{b o}$ when the maximum is achieved when $p_{2} \rightarrow p_{1}-\left(L_{2}-L_{1}\right) h_{b o}$.

When $p_{1} \leq c_{2}$, it is clear that the best option for supplier 2 is to avoid entering the market, i.e., $p_{2}=c_{2}$, since otherwise it would suffer a loss.

When $p_{1}>c_{2}$, it is clearly better to enter the market. There, the function $p_{2}^{0}$ can be characterized through the first-order condition: $p_{2}^{0}-c_{2}=\frac{\alpha_{2}\left(p_{1}-p_{2}^{0}\right)}{-\frac{d \alpha_{1}}{d \Delta}\left(p_{1}-p_{2}^{0}\right)}$. The left-hand side is increasing in $p_{2}^{0}$, and the right-hand side increasing in $p_{1}-p_{2}^{0}$. It is clear that, when $p_{1}$ increases, $p_{2}^{0}$ must increase, but not as much as $p_{1}$. Thus, the slope of $p_{2}^{0}$ is between 0 and 1 . Thus, there is $a_{2}$ such that for $p_{1} \leq c_{2}+a_{2}, p_{2}^{0}=p_{1}-\epsilon$ and for $p_{1}>c_{2}+a_{2}, p_{2}^{0}<p_{1}$.

In addition, $\Pi_{2}\left(p_{1}, p_{2}^{0}\left(p_{1}\right)\right)$ is increasing in $p_{1}$, and using the envelope theorem, and the first-order condition,

$$
\frac{d \Pi_{2}\left(p_{1}, p_{2}^{0}\left(p_{1}\right)\right)}{d p_{1}}=\left(p_{2}^{0}-c_{2}\right) \frac{d \alpha_{1}}{d \Delta}=\alpha_{2} \in[0,1] .
$$

This implies that $\Pi_{2}\left(p_{1}, p_{2}^{0}\left(p_{1}\right)\right) \geq \Pi_{2}\left(p_{1}, p_{1}-\left(L_{2}-L_{1}\right) h_{b o}\right)=p_{1}-\left(L_{2}-L_{1}\right) h_{b o}-c_{2}$ if and only if $p_{1} \leq c_{2}+e_{2}$ for some constant $e_{2}$. Thus, we have identified the four cases identified in the lemma. 


\section{A.3 Proof of Lemma 4}

Proof. If $\frac{1}{\alpha_{1}}$ is convex then $\alpha_{1} \frac{d^{2} \alpha_{1}}{d \Delta^{2}} \leq 2\left(\frac{d \alpha_{1}}{d \Delta}\right)^{2}$. Let $p_{1} \in\left(p_{2}, p_{2}+\left(L_{2}-L_{1}\right) h_{b o}\right)$ satisfy the first-order condition, i.e.,

$$
\frac{d \Pi_{1}}{d p_{1}}=\left(p_{1}-c_{1}\right) \frac{d \alpha_{1}}{d \Delta}+\alpha_{1}=0
$$

Then,

$$
\frac{d^{2} \Pi_{1}}{d p_{1}^{2}}=\left(p_{1}-c_{1}\right) \frac{d^{2} \alpha_{1}}{d \Delta^{2}}+2 \frac{d \alpha_{1}}{d \Delta}=\frac{-\alpha_{1} \frac{d^{2} \alpha_{1}}{d \Delta^{2}}+2\left(\frac{d \alpha_{1}}{d \Delta}\right)^{2}}{\frac{d \alpha_{1}}{d \Delta}} \leq 0 .
$$

Thus $\Pi_{1}$ must be increasing and then decreasing, and hence pseudo-concave.

\section{A.4 Proof of Lemma 5}

Proof. The proof follows exactly the proof of Lemma 3. The only difference arises from the fact that, if $\alpha_{1}$ is discontinuous at $\Delta=0$, then it may be optimal to set $p_{1}^{*}=p_{2}$ in order to push the slow supplier out of the market. This is true if and only if $\Pi_{1}\left(p_{2}, p_{2}\right)=p_{2}-c_{1} \geq \Pi_{1}\left(p_{1}^{0}\left(p_{2}\right), p_{2}\right)$. As before, $\Pi_{1}\left(p_{1}^{0}\left(p_{2}\right), p_{2}\right)$ increases with $p_{2}$ with slope smaller than 1 . Thus, there exists $c_{1}+e_{1}$ above which it is optimal to set $p_{1}^{*}\left(p_{2}\right)=p_{2}$, and below which $p_{1}^{*}\left(p_{2}\right)=p_{1}^{0}\left(p_{2}\right)$. This is a point of discontinuity in $p_{1}^{*}$. Note, that there is continuity when $\alpha_{1}$ is continuous.

In addition, when $c_{1}+a_{1}<p_{2} \leq c_{1}+e_{1}$, the first-order optimality condition is satisfied:

$$
\left(p_{1}^{0}-c_{1}\right) \frac{d \alpha_{1}}{d \Delta}+\alpha_{1}=0
$$

and hence, using the implicit function theorem,

$$
\frac{d p_{1}^{0}}{d p_{2}}=\frac{\left(\frac{d \alpha_{1}}{d \Delta}\right)^{2}-\alpha_{1} \frac{d^{2} \alpha_{1}}{d \Delta^{2}}}{2\left(\frac{d \alpha_{1}}{d \Delta}\right)^{2}-\alpha_{1} \frac{d^{2} \alpha_{1}}{d \Delta^{2}}} \leq 1
$$

\section{A.5 Proof of Lemma 6}

Proof. Clearly, when $p_{2}+\left(L_{2}-L_{1}\right) h_{b o} \leq c_{1}$, we have $p_{1}^{*}\left(p_{2}\right)=c_{1}$. Otherwise it is optimal for the fast supplier to enter the market, by setting $c_{1}<p_{1}<p_{2}+\left(L_{2}-L_{1}\right) h_{b o}$.

When $\frac{1}{\alpha_{1}}$ is concave, then any point satisfying the first-order conditions is a minimum of profit. As a consequence, the best price is either $p_{1}=p_{2}$ or $p_{1}=p_{2}+\left(L_{2}-L_{1}\right) h_{b o}-\epsilon$. We must hence compare two 
profits: $\Pi_{1}\left(p_{2}, p_{2}\right)=p_{2}-c_{1}$ and $\Pi_{1}\left(p_{2}+\left(L_{2}-L_{1}\right) h_{b o}-\epsilon, p_{2}\right)=\left(p_{2}+h_{b o}-\epsilon-c_{1}\right) \alpha_{1}\left(\left(L_{2}-L_{1}\right) h_{b o}-\epsilon\right)$. Thus, it is clear that there is $c_{1}+e_{1}$ above which $p_{1}^{*}\left(p_{2}\right)=p_{2}$, and below which $p_{1}^{*}\left(p_{2}\right)=p_{2}+\left(L_{2}-L_{1}\right) h_{b o}-\epsilon$.

\section{A.6 Proof of Theorem 1}

Proof. In order to find equilibrium, we must look for the intersection points of the curves characterized by Lemmas 3 and 5 or 6 (in which case $a_{1}=e_{1}$ ). Of course, the intersection can occur at three different points: either $p_{1}^{e q}=p_{2}^{e q}$, or $p_{1}^{e q}-p_{2}^{e q}=\left(L_{2}-L_{1}\right) h_{b o}$, or $0<p_{1}^{e q}-p_{2}^{e q}<\left(L_{2}-L_{1}\right) h_{b o}$. Clearly, the first two cases only happen when one of the suppliers drives the other out of the market. The third case takes place when $c_{1}+a_{1}<p_{2} \leq c_{1}+e_{1}$ and $c_{2}+a_{2}<p_{1} \leq c_{2}+e_{2}$, together with ensuring that the curves $p_{1}^{0}$ and $p_{2}^{0}$ intersect. Since in that segment the best-response functions are continuous, $\frac{d p_{1}^{*}}{d p_{2}} \leq 1$ and $\frac{d p_{2}^{*}}{d p_{1}} \in[0,1]$, we have an intersection if and only if $\lim _{\epsilon \rightarrow 0^{+}} p_{1}^{*}\left(c_{1}+e_{1}-\epsilon\right)-\left(c_{1}+e_{1}\right) \leq\left(c_{2}+e_{2}\right)-\lim _{\epsilon \rightarrow 0^{+}} p_{2}^{*}\left(c_{2}+e_{2}-\epsilon\right)$. It is clear that all these equilibria are unique.

\section{A.7 Proof of Lemma 7}

Proof. Let $\phi(x)=\frac{x}{f\left(\bar{F}^{-1}(x)\right)}$. If the demand's failure rate is non-decreasing, then this is a nondecreasing function, as

$$
\phi(\bar{F}(x))=\frac{\bar{F}(x)}{f(x)}
$$

is non-increasing in $x$. Then

$$
\frac{d \alpha_{1}}{d \Delta}=-\frac{h_{i n v}}{\bar{D}\left(h_{i n v}+\Delta\right)^{2}} \phi\left(\frac{h_{i n v}}{h_{i n v}+\Delta}\right)
$$

and hence

$$
\frac{d^{2} \alpha_{1}}{d \Delta^{2}}=2 \frac{h_{i n v}}{\bar{D}\left(h_{i n v}+\Delta\right)^{3}} \phi\left(\frac{h_{i n v}}{h_{i n v}+\Delta}\right)+\frac{h_{i n v}^{2}}{\bar{D}\left(h_{i n v}+\Delta\right)^{4}} \phi^{\prime}\left(\frac{h_{i n v}}{h_{i n v}+\Delta}\right) \geq 0
$$

\section{A.8 Proof of Theorem 2}

Proof. For this uniform distribution, $\alpha_{1}=\frac{D_{\max }-D_{\min }}{D_{\max }+D_{\min }}\left(\frac{h_{i n v}}{h_{i n v}+\Delta}\right)^{2}$ (see Example 1). Thus, $\alpha_{1}$ and $\frac{1}{\alpha_{1}}$ are convex. Let $\gamma=\frac{D_{\max }-D_{\min }}{D_{\max }+D_{\min }}$. 
First, the best-response function of supplier 1 is characterized by $a_{1}=-\infty$, for $p_{2} \leq c_{1}+e_{1}, p_{1}^{*}\left(p_{2}\right)=$ $2 c_{1}+h_{\text {inv }}-p_{2}$, which implies $\Delta=2 c_{1}-2 p_{2}+h_{\text {inv }} \geq 0$, and $e_{1}$ such that

$$
\frac{4 e_{1}\left(h_{i n v}-e_{1}\right)}{h_{i n v}^{2}}=\gamma
$$

(condition where the profit of setting $p_{1}=c_{1}+e_{1}$ and using $p_{1}=p_{1}^{0}\left(c_{1}+e_{1}\right)$ are equal), i.e.,

$$
e_{1}=h_{i n v}\left(\frac{1}{2}-\frac{1}{2} \sqrt{1-\gamma}\right)
$$

Second, the best-response function of supplier 2 is continuous, $p_{2}^{*}\left(p_{1}\right)=c_{2}$ for $p_{1} \leq c_{2}$, and increasing for $p_{1}>c_{2}$.

When $c_{2} \geq c_{1}+e_{1}$, we fall in the first case of Theorem 1. Notice that when $D_{\min }=0, c_{2} \geq c_{1}+e_{1}$ is equivalent to $\frac{c_{1}-c_{2}}{h_{\text {inv }}} \leq-\frac{1}{2}$. The second case never occurs, as $h_{b o}=\infty$. The third case corresponds to the equilibrium where the first-order conditions are satisfied:

$$
\begin{aligned}
\frac{p_{1}-c_{1}}{h_{i n v}} & =\frac{1}{2} \frac{h_{i n v}+\Delta}{h_{i n v}} \\
\frac{p_{2}-c_{2}}{h_{i n v}} & =\frac{1}{2 \gamma}\left(\frac{h_{i n v}+\Delta}{h_{i n v}}\right)^{3}-\frac{1}{2}\left(\frac{h_{i n v}+\Delta}{h_{i n v}}\right)
\end{aligned}
$$

By substracting the two equations, we can find the equilibrium $\Delta$ :

$$
\left(\frac{h_{i n v}+\Delta}{h_{i n v}}\right)^{3}=2 \gamma\left(1+\frac{c_{1}-c_{2}}{h_{i n v}}\right) .
$$

This has a solution such that $p_{2} \leq c_{1}+e_{1}$ or $\Delta \geq-2 e_{1}+h_{i n v}$ when

$$
\left(\frac{\left.2 h_{i n v}-2 e_{1}\right)}{h_{i n v}}\right)^{3} \leq 2 \gamma\left(1+\frac{c_{1}-c_{2}}{h_{i n v}}\right)
$$

i.e., after substitution, $(1+\sqrt{1-\gamma})^{3} \leq 2 \gamma\left(1+\frac{c_{1}-c_{2}}{h_{\text {inv }}}\right)$ or

$$
\frac{c_{1}-c_{2}}{h_{\text {inv }}} \geq \frac{(1+\sqrt{1-\gamma})^{3}}{2 \gamma}-1
$$

Notice that when $D_{\min }=0, \gamma=1$, and this is equivalent to $\frac{c_{1}-c_{2}}{h_{\text {inv }}} \geq-\frac{1}{2}$.

With this, we have identified the situation corresponding to case three in Theorem 1 . In any other case besides this and $c_{1}-c_{2} \leq-e_{1}$, there is no equilibrium. 


\section{A.9 Proof of Theorem 3}

Proof. For the exponential distribution distribution, $\alpha_{1}=\frac{h_{i n v}}{h_{i n v}+\Delta}$ (see Example 2). Thus, $\alpha_{1}$ and $\frac{1}{\alpha_{1}}$ are convex.

First, the best-response function of supplier 1 is characterized by $a_{1}=-\infty, e_{1}=h_{\text {inv }}$, and for $p_{2} \leq$ $c_{1}+e_{1}, p_{1}^{*}\left(p_{2}\right)=\infty$. Thus, there can be no equilibrium when $p_{2}<c_{1}+e_{1}$. When $p_{2}=c_{1}+e_{1}$, then any price $p_{1}$ above $p_{2}$ yields the same profit to supplier 1 .

Second, the best-response function of supplier 2 is continuous, $p_{2}^{*}\left(p_{1}\right)=c_{2}$ for $p_{1} \leq c_{2}$, and increasing for $p_{1}>c_{2}$.

Again, the first case of Theorem 1 corresponds to $c_{2} \geq c_{1}+e_{1}$. Since $h_{b o}=\infty$, the second case of the theorem never occurs. The third case corresponds to $p_{2}=c_{1}+e_{1}=c_{1}+h_{i n v}$. In that situation, the first-order condition of supplier 2 is satisfied, i.e.,

$$
\frac{p_{2}-c_{2}}{h_{i n v}}=\left(\frac{h_{i n v}+\Delta}{h_{i n v}}\right)^{2}-\left(\frac{h_{i n v}+\Delta}{h_{i n v}}\right)
$$

Substituting $p_{2}=c_{1}+h_{i n v}$ (required for supplier 1 to be in equilibrium, we have that

$$
\left(\frac{h_{i n v}+\Delta}{h_{i n v}}\right)^{2}-\frac{h_{i n v}+\Delta}{h_{i n v}}-1=\frac{c_{1}-c_{2}}{h_{i n v}}
$$

which yields

$$
\frac{\Delta}{h_{i n v}}=-\frac{1}{2}+\sqrt{\frac{c_{1}-c_{2}}{h_{i n v}}+\frac{5}{4}} .
$$

\section{A.10 Proof of Theorem 4}

Proof. Let $a=\frac{k}{h_{i n v}+h_{b o}}$. Since we are interested in situations with small $\Delta$, we consider that $\Delta<$ $h_{b o}\left(L_{2}-L_{1}\right)$ always.

We have

$$
\begin{aligned}
& \frac{d \Pi_{1}}{d p_{1}}=\left(p_{1}-c_{1}\right)\left(\frac{a}{2 \sqrt{\Delta}}\right)-(1-a \sqrt{\Delta})=\left(p_{2}-c_{1}\right)\left(\frac{a}{2 \sqrt{\Delta}}\right)-1+\frac{3}{2} a \sqrt{\Delta} \\
& \frac{d \Pi_{2}}{d p_{2}}=\left(p_{2}-c_{2}\right)\left(\frac{a}{2 \sqrt{\Delta}}\right)-a \sqrt{\Delta} .
\end{aligned}
$$

The best reaction for supplier 2 is always to set $p_{2}=c_{2}$ when $p_{1} \leq c_{2}$ and $p_{2}=\frac{1}{3} c_{2}+\frac{2}{3} p_{1}$, i.e., $\Delta=\frac{p_{1}-c_{2}}{3}$ when $p_{1}-c_{2} \leq \frac{3}{a^{2}}$, and $p_{2}=p_{1}-\frac{1}{a^{2}}$, otherwise.

On the other hand, supplier 1 may consider three strategies and select the best: 
(1) $p_{1}=p_{2}$, yielding $\Pi_{1}=p_{2}-c_{1}$;

(2) or find the unique price that satisfies the first-order condition

$$
\sqrt{p_{1}-p_{2}}=\frac{1}{3 a}+\frac{1}{3} \sqrt{\frac{1}{a^{2}}-3\left(p_{2}-c_{1}\right)},
$$

when the square root is well-defined and the corresponding market share is non-negative;

(3) or $p_{1}=c_{1}$, yielding $\Pi_{1}=0$.

Figure 8 shows the profit $\Pi_{1}$ for different parameters of $a$. We see that options (1) or $(2)$ are better depending on the values of $a$.

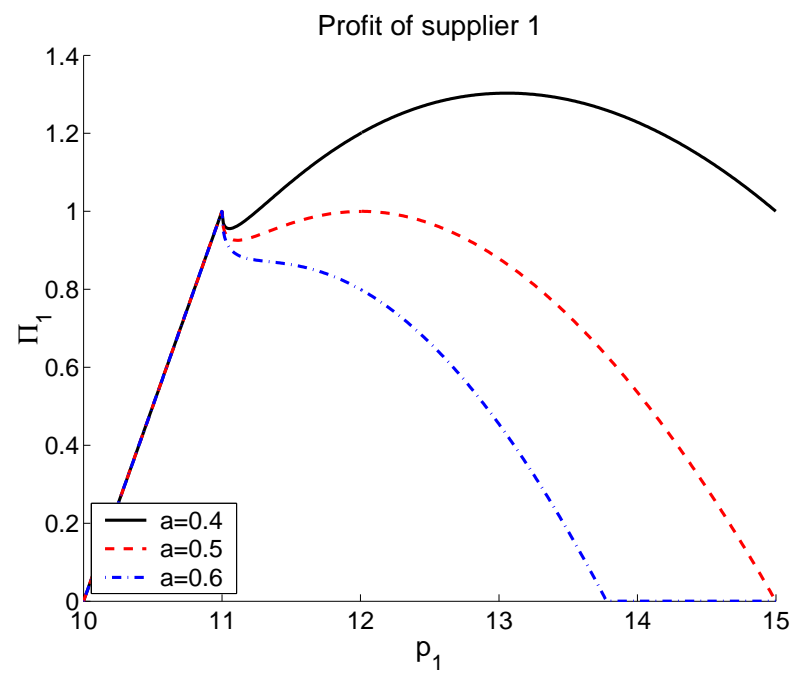

Figure 8: Plots of supplier 1 profit $\Pi_{1}$ as a function of $p_{1}$, for different parameters of $a$ and $c_{1}=10, p_{2}=11$. We observe that depending on the value of $a$, we may select $p_{1}^{*}=p_{2}$ or $p_{1}^{*}>p_{2}$, with a discontinuous jump at $a=0.5=\sqrt{\frac{1}{4\left(p_{2}-c_{1}\right)}}$.

It turns out that the second option is the best for supplier 1 if and only if

$$
-1 \leq a^{2}\left(p_{2}-c_{1}\right) \leq \frac{1}{4}
$$

The first option is to capture all the market by outbidding supplier 2 , and it corresponds to $p_{2}>c_{1}+\frac{1}{4 a^{2}}$. The third option corresponds to the situation where $p_{2}<c_{1}-\frac{1}{a^{2}}$. Figure 9 shows the best-response function of supplier 1 as a function to $p_{2}$. We clearly identify option (1) for large $p_{2}$. Then, after a jump, option (2) is the most advantageous. Finally, for smaller $p_{2}$, the best choice is $(3): p_{1}=c_{1}$. 


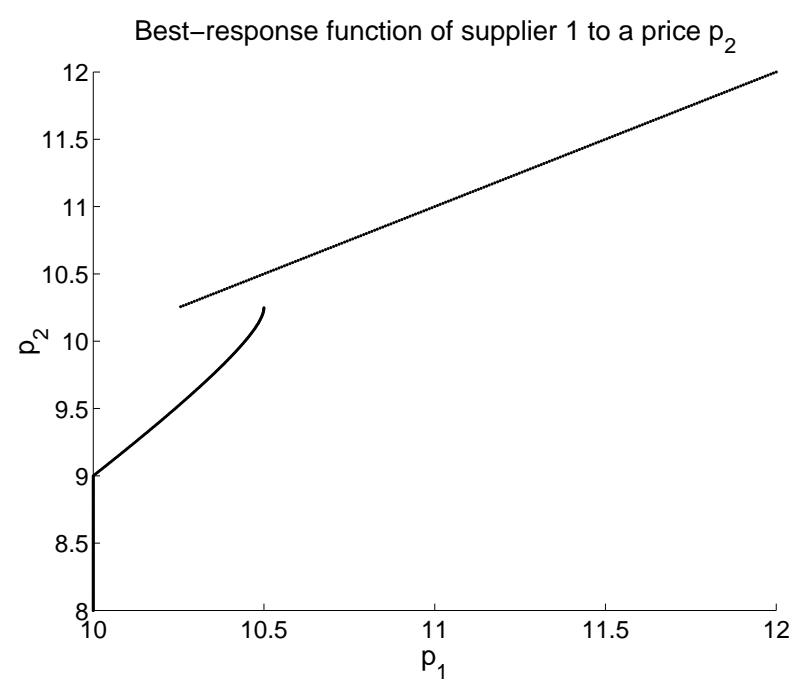

Figure 9: Best-response function $p_{1}^{*}\left(p_{2}\right)$ for supplier 1 when $a=1$ and $c_{1}=10$. Observe that for prices $p_{2}$ below 9 , the best response is to price at cost, with a resulting market share of 0 , since $\Delta \geq 1 / a^{2}$.

With the best-response functions, we compute the price equilibria of the system. We have four cases to consider.

When $a^{2}\left(c_{1}-c_{2}\right) \leq-\frac{1}{4}$, i.e., when the slow supplier has a cost $c_{2}$ significantly higher than $c_{1}$, it is not able to compete, since supplier 1 prices it out the market, by setting $p_{1}=c_{2}: p_{1}^{e q}=p_{2}^{e q}=c_{2}$. Thus, when $a$ is very large, we obtain a result similar to the asymmetric Bertrand competition model.

The second possible case is that no equilibrium exists: supplier 1 prices supplier 2 out of the market as soon as it enters, and when supplier 2 does not enter, supplier 1 sets a high price that leads 2 to entry. This happens when

$$
-\frac{1}{4} \leq a^{2}\left(c_{1}-c_{2}\right) \leq \frac{1}{4}
$$

which captures the case when both suppliers have the same cost.

Finally, the third possibility is that both suppliers obtain a positive market share. This occurs when

$$
\frac{1}{4} \leq a^{2}\left(c_{1}-c_{2}\right) \leq 3
$$

Here, the algebra yields that

$$
\begin{aligned}
& \sqrt{\Delta}=\frac{1}{5 a}+\sqrt{\frac{c_{1}-c_{2}}{5}+\frac{1}{25 a^{2}}} \\
& p_{2}=c_{2}+2 \Delta \\
& p_{1}=p_{2}+\Delta
\end{aligned}
$$


Finally, there is a last case when

$$
a^{2}\left(c_{1}-c_{2}\right) \geq 3
$$

Here, the cost difference is so large that supplier 1 cannot compete and is thrown out of the market completely. Thus, $p_{1}^{e q}=c_{1}$ and $p_{2}^{e q}=p_{1}^{e q}-\frac{1}{a^{2}}$.

\section{B Derivation of Market Share Approximation}

Below, we detail how we obtain the approximation presented in Section 7.

When $\Delta=0$, the double base-stock policy is optimal. Indeed, the base-stock level of supplier 1 is equal to the level of the newsboy, when supplier 1 is the only supplier and the demand is equal to $D_{t}-D_{\min }$. Thus, at $\Delta=0$, the optimality equation is

$$
\mathbb{P}\left(D_{t}+\ldots+D_{t+L_{1}-1}-L_{1} D_{\text {min }} \geq b_{1}\right)=\bar{F}_{L_{1}}\left(b_{1}\right)=\frac{h_{\text {inv }}}{h_{\text {inv }}+h_{b o}} .
$$

Also, $b_{2}=b_{1}+\left(L_{2}-L_{1}\right) D_{\min }$ is the optimal level for supplier 2. This guarantees that supplier 2 delivers exactly $D_{\min }$ at every period.

Consider now that how $\alpha_{1}$ varies for $\Delta>0$. Supplier 2 is used in small quantities above $D_{\text {min }}$. That is, using a base-stock policy, $b_{2}>b_{1}+\left(L_{2}-L_{1}\right) D_{\min }$. The amount to be shipped from supplier 2 is on average around $\left(b_{2}-b_{1}\right) /\left(L_{2}-L_{1}\right)$ per period. This is because the amount of stock in transit is equal to $b_{2}-Y_{t}^{L_{1}} \approx b_{2}-b_{1}$, and the transit time $L_{2}-L_{1}$.

In these conditions, the event of having an inventory position at supplier 1 larger than $b_{1}$ has a very small probability of happening. Our approximation is based on the assumption that two such events never occur in two consecutive periods. Intuitively, this occurs when the order placed at $t-\left(L_{2}-L_{1}\right)$ (due to arrive and used to serve demand at $\left.t+L_{1}\right)$ is larger than the demand $D_{t}$. Thus, the inventory position for supplier 1 is $b_{1}-D_{t}+Q_{t-\left(L_{2}-L_{1}\right)}^{2}>b_{1}$. Approximately, this occurs with probability equal to the stationary probability of $D_{t} \leq Q_{t-\left(L_{2}-L_{1}\right)}^{2}$, which is very small when $\Delta \approx 0$. In this situation, the order placed at supplier 1 is 0 , and the order placed at supplier 2 is $D_{t}$.

When, on the other hand, $D_{t}>Q_{t-\left(L_{2}-L_{1}\right)}^{2}$, then the order placed at supplier 1 is $D_{t}-Q_{t-\left(L_{2}-L_{1}\right)}^{2}$ and at supplier $2 Q_{t-\left(L_{2}-L_{1}\right)}^{2}$.

We can rewrite Equation (1), where we will take conditional expectations on $D_{t}>Q_{t-\left(L_{2}-L_{1}\right)}^{2}$ or 
$D_{t} \leq Q_{t-\left(L_{2}-L_{1}\right)}^{2}$. We count the purchasing cost at $t$ and the inventory cost taken at $t+L_{1}$.

$$
\begin{array}{r}
C=\quad p_{2} \bar{D}+\mathbb{P}\left(D_{t}>Q_{t-\left(L_{2}-L_{1}\right)}^{2}\right) \mathbb{E}_{D_{t}>Q_{t-\left(L_{2}-L_{1}\right)}^{2}}\left\{\begin{array}{c}
\Delta\left(D_{t}-Q_{t-\left(L_{2}-L_{1}\right)}^{2}\right) \\
+h_{\text {inv }}\left(b_{1}-D_{t+1}-\ldots-D_{t+L_{1}}\right)^{+} \\
-h_{b o}\left(b_{1}-D_{t+1}-\ldots-D_{t+L_{1}}\right)^{-}
\end{array}\right\} \\
\quad+\mathbb{P}\left(D_{t} \leq Q_{t-\left(L_{2}-L_{1}\right)}^{2}\right) \mathbb{E}_{D_{t} \leq Q_{t-\left(L_{2}-L_{1}\right)}^{2}}\left\{\begin{array}{c}
h_{\text {inv }}\left(b_{1}+Q_{t-\left(L_{2}-L_{1}\right)}^{2}-D_{t}-D_{t+1}-\ldots-D_{t+L_{1}}\right)^{+} \\
-h_{b o}\left(b_{1}+Q_{t-\left(L_{2}-L_{1}\right)}^{2}-D_{t}-D_{t+1}-\ldots-D_{t+L_{1}}\right)^{-}
\end{array}\right\}
\end{array}
$$

As we said, this can be approximated by

$$
\begin{aligned}
\tilde{C}= & p_{2} \bar{D}+\mathbb{P}\left(D_{t}>\frac{b_{2}-b_{1}}{L_{2}-L_{1}}\right) \mathbb{E}_{D_{t}>\frac{b_{2}-b_{1}}{L_{2}-L_{1}}}\left\{\begin{array}{c}
\Delta\left(D_{t}-\frac{b_{2}-b_{1}}{L_{2}-L_{1}}\right) \\
+h_{i n v}\left(b_{1}-D_{t+1}-\ldots-D_{t+L_{1}}\right)^{+} \\
-h_{b o}\left(b_{1}-D_{t+1}-\ldots-D_{t+L_{1}}\right)^{-}
\end{array}\right\} \\
+ & \mathbb{P}\left(D_{t} \leq \frac{b_{2}-b_{1}}{L_{2}-L_{1}}\right) \mathbb{E}_{D_{t} \leq \frac{b_{2}-b_{1}}{L_{2}-L_{1}}}\left\{\begin{array}{c}
+h_{i n v}\left(b_{1}+\frac{b_{2}-b_{1}}{L_{2}-L_{1}}-D_{t}-D_{t+1}-\ldots-D_{t+L_{1}}\right)^{+} \\
-h_{b o}\left(b_{1}+\frac{b_{2}-b_{1}}{L_{2}-L_{1}}-D_{t}-D_{t+1}-\ldots-D_{t+L_{1}}\right)^{-}
\end{array}\right\}
\end{aligned}
$$

We can optimize $\tilde{C}$ over $b_{1}$ and $b_{2}-b_{1}$ separately.

$$
\begin{aligned}
0= & \frac{d \tilde{C}}{d\left(b_{2}-b_{1}\right)}\left(L_{2}-L_{1}\right) \\
= & -\Delta \mathbb{P}\left(D_{t}>\frac{b_{2}-b_{1}}{L_{2}-L_{1}}\right) \\
& +\mathbb{P}\left(D_{t} \leq \frac{b_{2}-b_{1}}{L_{2}-L_{1}}\right) \mathbb{E}_{D_{t} \leq \frac{b_{2}-b_{1}}{L_{2}-L_{1}}}\left\{h_{i n v}-\left(h_{i n v}+h_{b o}\right) \overline{F_{L_{1}}}\left(b_{1}+\frac{b_{2}-b_{1}}{L_{2}-L_{1}}-D_{t}\right)\right\}
\end{aligned}
$$

and

$$
\begin{aligned}
0= & \frac{d \tilde{C}}{d b_{1}} \\
= & \mathbb{P}\left(D_{t}>\frac{b_{2}-b_{1}}{L_{2}-L_{1}}\right)\left\{h_{i n v}-\left(h_{i n v}+h_{b o}\right) \overline{F_{L_{1}}}\left(b_{1}\right)\right\} \\
& +\mathbb{P}\left(D_{t} \leq \frac{b_{2}-b_{1}}{L_{2}-L_{1}}\right) \mathbb{E}_{D_{t} \leq \frac{b_{2}-b_{1}}{L_{2}-L_{1}}}\left\{h_{i n v}-\left(h_{i n v}+h_{b o}\right) \overline{F_{L_{1}}}\left(b_{1}+\frac{b_{2}-b_{1}}{L_{2}-L_{1}}-D_{t}\right)\right\}
\end{aligned}
$$

We can combine both equations and obtain, on the one hand, $\overline{F_{L_{1}}}\left(b_{1}\right)=\frac{h_{i n v}+\Delta}{h_{i n v}+h_{b o}}$, and, on the other hand,

$$
\begin{aligned}
\Delta & =\mathbb{P}\left(D_{t} \leq \frac{b_{2}-b_{1}}{L_{2}-L_{1}}\right) \mathbb{E}_{D_{t} \leq \frac{b_{2}-b_{1}}{L_{2}-L_{1}}}\left\{h_{i n v}+\Delta-\left(h_{i n v}+h_{b o}\right) \overline{F_{L_{1}}}\left(b_{1}+\frac{b_{2}-b_{1}}{L_{2}-L_{1}}-D_{t}\right)\right\} \\
& =\left(h_{i n v}+h_{b o}\right) \mathbb{P}\left(D_{t} \leq \frac{b_{2}-b_{1}}{L_{2}-L_{1}}\right) \mathbb{E}_{D_{t} \leq \frac{b_{2}-b_{1}}{L_{2}-L_{1}}}\left\{\overline{F_{L_{1}}}\left(b_{1}\right)-\overline{F_{L_{1}}}\left(b_{1}+\frac{b_{2}-b_{1}}{L_{2}-L_{1}}-D_{t}\right)\right\} \\
& =\left(h_{i n v}+h_{b o}\right) \mathbb{P}\left(b_{1} \leq D_{t+1}+\ldots+D_{t+L_{1}} \leq b_{1}+\frac{b_{2}-b_{1}}{L_{2}-L_{1}}-D_{t}\right)
\end{aligned}
$$

